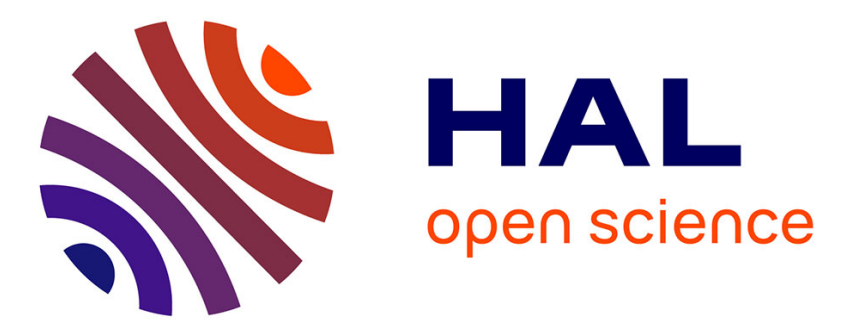

\title{
Geometric Particle-in-Cell methods for the Vlasov-Maxwell equations with spin effects
}

Nicolas Crouseilles, Paul-Antoine Hervieux, Yingzhe Li, Giovanni Manfredi, Yajuan Sun

\section{- To cite this version:}

Nicolas Crouseilles, Paul-Antoine Hervieux, Yingzhe Li, Giovanni Manfredi, Yajuan Sun. Geometric Particle-in-Cell methods for the Vlasov-Maxwell equations with spin effects. Journal of Plasma Physics, 2021, 87 (3), pp.article n 825870301. 10.1017/S0022377821000532 . hal-03148534v2

\section{HAL Id: hal-03148534 \\ https://hal.inria.fr/hal-03148534v2}

Submitted on 2 Jun 2021

HAL is a multi-disciplinary open access archive for the deposit and dissemination of scientific research documents, whether they are published or not. The documents may come from teaching and research institutions in France or abroad, or from public or private research centers.
L'archive ouverte pluridisciplinaire HAL, est destinée au dépôt et à la diffusion de documents scientifiques de niveau recherche, publiés ou non, émanant des établissements d'enseignement et de recherche français ou étrangers, des laboratoires publics ou privés. 


\title{
Geometric Particle-in-Cell methods for the Vlasov-Maxwell equations with spin effects
}

\author{
Nicolas Crouseilles ${ }^{1} \dagger$, Paul-Antoine Hervieux ${ }^{2}$, Yingzhe Li $^{3}$, Giovanni \\ Manfredi ${ }^{2} \ddagger$, and Yajuan $\mathrm{Sun}^{4}$ \\ ${ }^{1}$ Univ Rennes, Inria Rennes Bretagne Atlantique (Mingus team) and IRMAR UMR CNRS \\ 6625, F-35042 Rennes, France \\ ${ }^{2}$ Université de Strasbourg, CNRS, Institut de Physique et Chimie des Matériaux de \\ Strasbourg, UMR 7504, F-67000 Strasbourg, France \\ ${ }^{3}$ Max Planck Institute for Plasma Physics, Boltzmannstrasse 2, 85748 Garching, Germany \\ ${ }^{4}$ Academy of Mathematics and Systems Science, Chinese Academy of Sciences, 100190 \\ University of Chinese Academy of Sciences, 100049 Beijing, PR China
}

(Received xx; revised $\mathrm{xx}$; accepted $\mathrm{xx}$ )

We propose a numerical scheme to solve the semiclassical Vlasov-Maxwell equations for electrons with spin. The electron gas is described by a distribution function $f(t, \mathbf{x}, \mathbf{p}, \mathbf{s})$ that evolves in an extended 9-dimensional phase space $(\mathbf{x}, \mathbf{p}, \mathbf{s})$, where $\mathbf{s}$ represents the spin vector. Using suitable approximations and symmetries, the extended phase space can be reduced to 5D: $\left(x, p_{x}, \mathbf{s}\right)$. It can be shown that the spin Vlasov-Maxwell equations enjoy a Hamiltonian structure that motivates the use of the recently developed geometric particle-in-cell (PIC) methods. Here, the geometric PIC approach is generalized to the case of electrons with spin. Total energy conservation is very well satisfied, with a relative error below $0.05 \%$. As a relevant example, we study the stimulated Raman scattering of an electromagnetic wave interacting with an underdense plasma, where the electrons are partially or fully spin polarized. It is shown that the Raman instability is very effective in destroying the electron polarization.

\section{Introduction}

The interaction of an electromagnetic pulse with the electronic spins in metallic or magnetic nano-objects has been the object of intense investigations, both theoretical and experimental, over the past few decades (Bigot \& Vomir 2013). It is well known that spin effects can play a decisive role in nanometric systems, most notably the Zeeman effect (coupling the spin to an external magnetic field) and the spin-orbit interaction (coupling the spin to the orbital motion of the electron). In particular, the ultrafast loss of magnetization observed in ferromagnetic nano-objects (Beaurepaire et al. 1996; Bigot et al. 2009 Bigot \& Vomir 2013) has been linked to various spin-dependent mechanisms, although its fundamental origin is still being debated. Metallic and magnetic nano-objects have many potential technological applications, including to biology and medicine, where they can be used to absorb electromagnetic energy and release it as heat in order to destroy cancer cells (hyperthermia) (Mehdaoui et al. 2013). In plasma physics, polarized electron beams of high spin polarization can now be created and precisely manipulated in the laboratory (Wu et al. 2019, 2020, Nie et al. 2021). Recently, numerical simulations of spin-polarized electrons beams interacting with strong laser pulses were performed by Wen et al. (2017, 2019).

$\dagger$ Email address for correspondence: nicolas.crouseilles@inria.fr

$\ddagger$ Email address for correspondence: giovanni.manfredi@ipcms.unistra.fr 
From a fundamental point of view, modelling the $N$-body dynamics of a system of particles possessing both charge and spin, represents an ambitious theoretical and computational challenge. Several approaches have been proposed in recent decades to tackle this difficult problem, relying either on hydrodynamic equations (Moldabekov et al. 2018) or on wave-function-based methods such as density functional theory (DFT) (Krieger et al. 2015).

Here, we will consider a possible alternative that relies on the use of the phase space models inspired from classical plasma physics, for which the system is governed by a probability distribution function that evolves according to a kinetic equation (Manfredi et al. 2019). The quantum equivalent of the classical distribution function can be obtained from Wigner's phase space representation of quantum mechanics. In this representation, which is completely equivalent to the more standard Schrödinger or Heisenberg representations, the state of a quantum system can be represented by a function $f$ of the phase space variables. The Wigner function evolves according to an integro-differential equation that reduces, in the classical limit, to the Vlasov equation. The Wigner function can be used to compute averages as in the classical case, but should not be considered as a proper probability distribution, because it can take negative values.

If one retains the spin degrees of freedom, the Wigner distribution function becomes a $2 \times 2$ matrix, whose elements represent the spin-up and spin-down components, as well as entangled states (Arnold \& Steinrück 1989). The corresponding semiclassical matrix spin Vlasov equation, coupled to Maxwell's equations, constitutes a viable mean-field model where the electron orbital motion is treated classically, while the spin is a fully quantum variable (Hurst et al. 2017, 2014). This approach was recently used to study the generation of spin currents in ferromagnetic thin films (Hurst et al. 2018).

A different, but equivalent, approach consists in defining a scalar distribution function that evolves in an extended phase space $(\mathbf{x}, \mathbf{p}, \mathbf{s})$, where the spin is a further variable (in addition to the position and the momentum) described by a vector on the unit-radius sphere (Marklund et al. 2010; Asenjo et al. 2012 ; Zamanian et al. 2010 a b) . The geometric structure of this scalar spin Vlasov model has been highlighted in Marklund \& Morrison (2011).

Both approaches (extended phase space and matrix Wigner function) are mathematically equivalent. From a computational point of view, the extended phase space method is more easily simulated using particle-in-cell (PIC) methods, because the corresponding distribution function is transported along classical trajectories in the extended phase space. In contrast, the matrix Wigner function method is more naturally amenable to grid-based Vlasov codes, because the corresponding distribution function only depends on the six variables of the ordinary phase space.

In this work, we will develop and validate a PIC code for the self-consistent spin Vlasov-Maxwell (spin-VM) equations in the extended phase space. The adopted model is semiclassical, in the sense that the orbital electron motion is treated in a classical fashion, while the spin dynamics is fully quantum. The electron spin intervenes in the dynamics via the Zeeman effect and a precession term, which are due to the coupling of the spin with a self-consistent or external magnetic field. These are the first non-relativistic corrections to the spin-less dynamics.

Despite the plentiful theoretical developments, numerical works on the simulation of spin effects in plasmas are relatively scarce. Cowley et al. (1986) were perhaps the first authors to perform computer simulations of polarized electrons in a plasma using a semiclassical kinetic approach. More recently, Brodin et al. (2013) developed a PIC code accounting for the magnetic dipole force and the magnetization currents associated with the electron spin. This work does not follow the extended phase space approach 
adopted here, but rather considers two separate spin-up and spin-down populations for the electrons, which is less general (this is know as the collinear approximation in condensed matter physics). In a similar context, Tonge et al. (2004) and Dauger et al. (2005) have extended classical PIC methods to the quantum regime (but without spin) using an approach based on Feynman path integrals. Very recently, PIC simulation methods for particles with spin were developed and validated by Li et al. (2021) for applications to laser-plasma interactions.

Further, in order to reduce the dimensionality of the problem, we consider a simplified model widely used to study laser-plasma interactions (Ghizzo et al. 1990, Huot et al. 2003, Li et al. $2019 b$ ) and assume that all quantities depend spatially only on the longitudinal co-ordinate $x$ (the direction of propagation of the incident electromagnetic wave). We also make the hypothesis that the electron distribution function can be written in the form:

$$
F(t, x, \mathbf{p}, \mathbf{s})=\delta\left(\mathbf{p}_{\perp}-e \mathbf{A}_{\perp}\right) f\left(t, x, p_{x}, \mathbf{s}\right),
$$

where $f\left(t, x, p_{x}, \mathbf{s}\right)=\int F(t, x, \mathbf{p}, \mathbf{s}) \mathrm{d} \mathbf{p}_{\perp}$ and $\mathbf{p}=\left(p_{x}, \mathbf{p}_{\perp}\right)=\left(p_{x}, p_{y}, p_{z}\right)$. Equation (1.1) is equivalent to stating that the electrons are cold in the perpendicular plane. Note that this ansatz is exact, in the sense that, if satisfied by the initial condition, then it is preserved along the time evolution of the Vlasov-Maxwell equations. By using this approximation, the extended phase space is reduced from 9 dimensions to 5 (one in space, one in velocity, and three for the spin).

The Poisson structure proposed in Marklund \& Morrison (2011) lays the bases to construct a geometric PIC method (Qin et al. $\mid 2015$ : Xiao et al. 2015 ; Kraus et al. 2017, He et al. 2016, Morrison 2017, Burby|2017) for the spin-VM equations. The geometric PIC method adopted here is based on a compatible finite-element approximation of the electromagnetic fields, combined with a particle approximation of the distribution function. Following Kraus et al. (2017), we have chosen spline spaces, which is a family of compatible finite elements in the sense that this approach enables to naturally derive discrete versions of the differential operators. The so-obtained finite-dimensional system of ordinary differential equations (ODEs) possesses a non-canonical Poisson structure. Subsequently, one has to implement a time discretization for this large ODE system. This is performed by decomposing the discrete Hamiltonian into several subsystems (Crouseilles et al. 2015: Li et al. 2019a: He et al. 2015) and using a time splitting method. It turns out that each of the subsystem can be solved exactly in time, meaning that their composition is still a Poisson map and high-order splitting schemes can thus be easily constructed.

The resulting spin-PIC code is tested on a well-studied problem in the physics of laser-plasma interactions, namely the stimulated Raman scattering (SRS). SRS describes the decay of an incident electromagnetic (em) wave into a scattered em wave and a plasma (Langmuir) wave, through a parametric instability. First, we benchmark our code against analytical results and former simulations carried out in the spin-less regime. Then we exploit the full spin Vlasov-Maxwell model to study the effect of the electron spin polarization on the Raman instability.

The rest of the paper is organized as follows. In section 2 the spin Vlasov models are presented together with their Poisson bracket formulation. In section 3 , the geometric electromagnetic PIC (GEMPIC) framework (Kraus et al. 2017) is recalled and extended to our spin context. In section 4 , the Hamiltonian time-splitting technique is described, and finally in section 5 several numerical simulations of the spin-dependent Raman scattering are presented and discussed. Finally, we draw our conclusions in section 6 . 


\section{Spin Vlasov-Maxwell equations}

\subsection{General formalism}

Here, we recall the basics of the spin Vlasov-Maxwell system in the extended phase space (Marklund \& Morrison|2011;: Marklund et al.|2010, Zamanian et al.|2010a: Manfredi et al. 2019), satisfied by the scalar electron distribution function:

$$
f:(t, \mathbf{x}, \mathbf{p}, \mathbf{s}) \in \mathbb{R}_{+} \times \mathbb{R}^{3} \times \mathbb{R}^{3} \times \mathbb{R}^{3} \mapsto f(t, \mathbf{x}, \mathbf{p}, \mathbf{s}) \in \mathbb{R},
$$

and the self-consistent electromagnetic fields $(\mathbf{E}, \mathbf{B}):(t, \mathbf{x}) \mapsto(\mathbf{E}, \mathbf{B})(t, \mathbf{x}) \in \mathbb{R}^{3} \times \mathbb{R}^{3}$. The spin Vlasov-Maxwell equations read as

$$
\begin{aligned}
\frac{\partial f}{\partial t} & +\frac{\mathbf{p}}{m} \cdot \nabla f+\left[q\left(\mathbf{E}+\frac{\mathbf{p}}{m} \times \mathbf{B}\right)+\mu_{e} \nabla(\mathbf{s} \cdot \mathbf{B})\right] \cdot \frac{\partial f}{\partial \mathbf{p}}+\frac{2 \mu_{e}}{\hbar}(\mathbf{s} \times \mathbf{B}) \cdot \frac{\partial f}{\partial \mathbf{s}}=0 \\
\epsilon_{0} \mu_{0} \frac{\partial \mathbf{E}}{\partial t} & =\nabla \times \mathbf{B}-\mu_{0} \mathbf{J} \\
\frac{\partial \mathbf{B}}{\partial t} & =-\nabla \times \mathbf{E} \\
\nabla \cdot \mathbf{E} & =\frac{\rho-q n_{0}}{\epsilon_{0}} \\
\nabla \cdot \mathbf{B} & =0
\end{aligned}
$$

In the original paper on the scalar spin-Vlasov equation (Zamanian et al. $\mid 2010 a$ ), one further term was present, which has the form of a modified quantum magnetic dipole term:

$$
\mu_{e} \nabla\left(\mathbf{B} \cdot \frac{\partial}{\partial \mathbf{s}}\right) \cdot \frac{\partial f}{\partial \mathbf{p}} .
$$

In subsequent works by the same authors, this term was often ignored because of the semiclassical approximation made there (Brodin et al. 2008; Brodin \& Stefan 2013, Brodin et al. 2011). This can be justified by assuming that variations of $f$ in spin space are of moderate size (for a more detailed discussion, see (Zamanian et al. 2010a, Brodin et al. 2011)). It should also be pointed out that this modified quantum dipole term contains derivatives in both real and spin space, thus making the PIC algorithm much more involved. For these reasons, such extra term is neglected in the present developments and will be left to future works on this topic.

The coupling between the Vlasov and Maxwell equations is ensured by the current and the density $(\mathbf{J}, \rho):(t, \mathbf{x}) \mapsto(\mathbf{J}, \rho)(t, \mathbf{x}) \in \mathbb{R}^{3} \times \mathbb{R}_{+}$, defined respectively as

$$
\mathbf{J}=q \int_{\mathbb{R}^{6}} \mathbf{p} f \mathrm{~d} \mathbf{p} \mathrm{d} \mathbf{s}+\mu_{e} \nabla \times \int_{\mathbb{R}^{6}} \mathbf{s} f \mathrm{~d} \mathbf{p} \mathrm{d} \mathbf{s}, \quad \rho=q \int_{\mathbb{R}^{6}} f \mathrm{~d} \mathbf{p} \mathrm{d} \mathbf{s} .
$$

Here, $q=-e$ is the electron charge, $\mu_{e}=\frac{q \hbar}{2 m}$ is the Bohr magneton, $\hbar$ the Planck constant, $m$ the electron mass, $n_{0}$ the fixed ion density, and $\epsilon_{0}, \mu_{0}$ denote the permittivity and the permeability of vacuum (which satisfy $\epsilon_{0} \mu_{0}=c^{-2}$, with $c$ being the speed of light). We further note that, in this model, the spin vector $\mathbf{s}$ is dimensionless and has fixed length, ie $|\mathbf{s}|=1$ or $\mathbf{s} \in \mathbb{S}^{2}$. Hence, the effective phase space is actually 8D. However, in order to preserve the geometric structure that will be used in the forthcoming sections, we will consider that $\mathbf{s} \in \mathbb{R}^{3}$.

In the above Vlasov equation (2.1), the first three terms are the standard terms present in the spin-less case, the next term $[\nabla(\mathbf{s} \cdot \mathbf{B})]$ is the Zeeman effect, and the last term $(\mathbf{s} \times \mathbf{B})$ represents the spin precession in the magnetic field. In equation 2.2$)$, the second 
term in the current represents the curl of the internal magnetization due to the electron spin. Further details can be found in Manfredi et al. (2019).

We shall use normalized units, denoted by a tilde and defined as follows:

$$
\frac{t}{1 / \omega_{p}}=\tilde{t}, \frac{\mathbf{x}}{\lambda}=\tilde{\mathbf{x}}, \frac{\mathbf{p}}{m c}=\tilde{\mathbf{p}}, \frac{f}{n_{0} / c^{3}}=\tilde{f}, \frac{\mathbf{E}}{\omega_{p} c m /|q|}=\tilde{\mathbf{E}}, \frac{\mathbf{B}}{\omega_{p} m /|q|}=\tilde{\mathbf{B}},
$$

where we introduced the electron plasma frequency, the plasma skin depth, and the scaled Planck constant:

$$
\omega_{p}=\sqrt{\frac{n_{0} q^{2}}{m \epsilon_{0}}}, \quad \lambda=\frac{c}{\omega_{p}}, \quad \mathfrak{h}=\frac{\hbar \omega_{p}}{2 m c^{2}} .
$$

The dimensionless version of 2.1 reads as

$$
\begin{aligned}
\frac{\partial f}{\partial t} & +\mathbf{p} \cdot \nabla f+[(\mathbf{E}+\mathbf{p} \times \mathbf{B})+\mathfrak{h} \nabla(\mathbf{s} \cdot \mathbf{B})] \cdot \frac{\partial f}{\partial \mathbf{p}}+(\mathbf{s} \times \mathbf{B}) \cdot \frac{\partial f}{\partial \mathbf{s}}=0, \\
\frac{\partial \mathbf{E}}{\partial t} & =\nabla \times \mathbf{B}-\int_{\mathbb{R}^{6}} \mathbf{p} f \mathrm{~d} \mathbf{p d} \mathbf{s}+\mathfrak{h} \nabla \times \int_{\mathbb{R}^{6}} \mathbf{s} f \mathrm{~d} \mathbf{p d} \mathbf{s}, \\
\frac{\partial \mathbf{B}}{\partial t} & =-\nabla \times \mathbf{E}, \\
\nabla \cdot \mathbf{E} & =\int_{\mathbb{R}^{6}} f \mathrm{~d} \mathbf{p d} \mathbf{s}-1, \\
\nabla \cdot \mathbf{B} & =0,
\end{aligned}
$$

where we removed the tilde in order to simplify the notation. The above dimensionless version of the spin Vlasov-Maxwell model will be used throughout this work.

It has been shown in Marklund \& Morrison (2011) that the equations (2.3) enjoy a noncanonical Poisson structure that enables us to rewrite this complex system in a more compact way. Let us denote $\mathcal{M}=\{(f, \mathbf{E}, \mathbf{B}) \mid \nabla \cdot \mathbf{B}=0\}$ the infinite dimensional manifold. The system (2.3) can be expressed using the Poisson bracket introduced in (Marklund \& Morrison 2011). For two functionals $(\mathcal{F}, \mathcal{G})$ acting on $\mathcal{M}$, we consider the following Poisson bracket, which is the sum of the Vlasov-Maxwell bracket (Marsden \& Weinstein 1982) and a bracket describing spin effects:

$$
\begin{aligned}
\{\mathcal{F}, \mathcal{G}\} & =\{\mathcal{F}, \mathcal{G}\}_{V M}+\{\mathcal{F}, \mathcal{G}\}_{s}, \quad \text { with } \\
\{\mathcal{F}, \mathcal{G}\}_{V M} & =\int_{\mathbb{R}^{9}} f\left[\frac{\delta \mathcal{F}}{\delta f}, \frac{\delta \mathcal{G}}{\delta f}\right]_{\mathbf{x p}} \mathrm{d} \mathbf{x} \mathrm{d} \mathbf{p} \mathrm{d} \mathbf{s} \\
& +\int_{\mathbb{R}^{9}}\left(\frac{\delta \mathcal{F}}{\delta \mathbf{E}} \cdot \frac{\partial f}{\partial \mathbf{p}} \frac{\delta \mathcal{G}}{\delta f}-\frac{\delta \mathcal{G}}{\delta \mathbf{E}} \cdot \frac{\partial f}{\partial \mathbf{p}} \frac{\delta \mathcal{F}}{\delta f}\right) \mathrm{d} \mathbf{x} \mathrm{d} \mathbf{p d} \mathbf{s} \\
& +\int_{\mathbb{R}^{3}}\left(\frac{\delta \mathcal{F}}{\delta \mathbf{E}} \cdot\left(\nabla \times \frac{\delta \mathcal{G}}{\delta \mathbf{B}}\right)-\frac{\delta \mathcal{G}}{\delta \mathbf{E}} \cdot\left(\nabla \times \frac{\delta \mathcal{F}}{\delta \mathbf{B}}\right)\right) \mathrm{d} \mathbf{x} \\
& +\int_{\mathbb{R}^{9}} f \mathbf{B} \cdot\left(\frac{\partial}{\partial \mathbf{p}} \frac{\delta \mathcal{F}}{\delta f} \times \frac{\partial}{\partial \mathbf{p}} \frac{\delta \mathcal{G}}{\delta f}\right) \mathrm{d} \mathbf{x} \mathrm{d} \mathbf{p} \mathrm{d} \mathbf{s}, \\
\{\mathcal{F}, \mathcal{G}\}_{s} & =\frac{1}{\mathfrak{h}} \int_{\mathbb{R}^{9}} f \mathbf{s} \cdot\left(\frac{\partial}{\partial \mathbf{s}} \frac{\delta \mathcal{F}}{\delta f} \times \frac{\partial}{\partial \mathbf{s}} \frac{\delta \mathcal{G}}{\delta f}\right) \mathrm{d} \mathbf{x} \mathrm{d} \mathbf{p} \mathrm{d} \mathbf{s} .
\end{aligned}
$$

Here, $[\cdot, \cdot]_{\mathbf{x p}}$ denotes the Lie bracket for two functions of $(\mathbf{x}, \mathbf{p})$. It was proven in Marklund \& Morrison (2011) that the bracket 2.4 is indeed a Poisson bracket. With the Hamiltonian functional defined as follows:

$$
\mathcal{H}(f, \mathbf{E}, \mathbf{B})=\frac{1}{2} \int_{\mathbb{R}^{9}}|\mathbf{p}|^{2} f \mathrm{~d} \mathbf{x} \mathrm{d} \mathbf{p} \mathrm{d} \mathbf{s}+\mathfrak{h} \int_{\mathbb{R}^{9}} \mathbf{s} \cdot \mathbf{B} f \mathrm{~d} \mathbf{x} \mathrm{d} \mathbf{p} \mathrm{d} \mathbf{s}+\frac{1}{2} \int_{\mathbb{R}^{3}}\left(|\mathbf{E}|^{2}+|\mathbf{B}|^{2}\right) \mathrm{d} \mathbf{x},
$$


the spin Vlasov-Maxwell system (2.3) can be written in a compact way, using $\mathcal{Z}:=$ $(f, \mathbf{E}, \mathbf{B}) \in \mathcal{M}$ :

$$
\frac{\partial \mathcal{Z}}{\partial t}=\{\mathcal{Z}, \mathcal{H}\}
$$

where $\{\cdot, \cdot\}$ is given by 2.4 . This system is supplemented with an initial condition $\mathcal{Z}(t=0)=\mathcal{Z}_{0}$.

\subsection{Reduced spin Vlasov-Maxwell equations}

Here, following Ghizzo et al. (1990), we derive a reduced spin Vlasov-Maxwell model by considering the case of a plasma interacting with an electromagnetic wave propagating in the longitudinal $x$ direction and assuming that all fields depend spatially on $x$ only. Choosing the Coulomb gauge $\nabla \cdot \mathbf{A}=0$, the vector potential $\mathbf{A}$ lies in the perpendicular (transverse) plane, ie $\mathbf{A}=\left(0, A_{y}, A_{z}\right)=\left(0, \mathbf{A}_{\perp}\right)$. Using $\mathbf{E}=-\nabla \phi-\partial_{t} \mathbf{A}$, we then obtain, using the notations $\mathbf{E}=\left(E_{x}, E_{y}, E_{z}\right)=\left(E_{x}, \mathbf{E}_{\perp}\right)$ :

$$
\mathbf{E}_{\perp}=-\partial_{t} \mathbf{A}_{\perp} \text { and } E_{x}=-\partial_{x} \phi .
$$

This prescription implies that the electric field is mainly electromagnetic in the transverse plane and mainly electrostatic in the longitudinal direction.

We then consider a distribution function of the form: $\delta\left(\mathbf{p}_{\perp}-\mathbf{A}_{\perp}\right) f\left(t, x, p_{x}, \mathbf{s}\right)$, where $\mathbf{p}=\left(p_{x}, p_{y}, p_{z}\right)=\left(p_{x}, \mathbf{p}_{\perp}\right)$ is the linear momentum and $\mathbf{p}-\mathbf{A}$ is the canonical momentum. The above assumption on the distribution function is tantamount to prescribing that the plasma is cold in the transverse plane. After integration with respect to $\mathbf{p}_{\perp}$, the relevant extended phase space is reduced to $5 \mathrm{D}$ (instead of 9D for the general case). The longitudinal variable $p_{x}$ will be simply denoted by $p$ in the sequel.

The reduced spin Vlasov-Maxwell system satisfied by the electron distribution function $f(x, p, \mathbf{s}, t)\left(x, p \in \mathbb{R}\right.$ and $\left.\mathbf{s} \in \mathbb{R}^{3}\right)$, the electric field $\mathbf{E}(t, x)=\left(E_{x}, \mathbf{E}_{\perp}\right)(t, x)=$ $\left(E_{x}, E_{y}, E_{z}\right)(t, x)$, and the vector potential $\mathbf{A}(t, x)=\left(A_{x}, \mathbf{A}_{\perp}\right)(t, x)=\left(0, A_{y}, A_{z}\right)(t, x)$ can be written as:

$$
\begin{aligned}
& \frac{\partial f}{\partial t}+p \frac{\partial f}{\partial x}+\left[E_{x}-\mathfrak{h} s_{y} \frac{\partial^{2} A_{z}}{\partial x^{2}}+\mathfrak{h} s_{z} \frac{\partial^{2} A_{y}}{\partial x^{2}}-\mathbf{A}_{\perp} \cdot \frac{\partial \mathbf{A}_{\perp}}{\partial x}\right] \frac{\partial f}{\partial p}+(\mathbf{s} \times \mathbf{B}) \cdot \frac{\partial f}{\partial \mathbf{s}}=0 \\
& \mathbf{B}=\nabla \times \mathbf{A}=\left[0, \frac{\partial A_{z}}{\partial x},-\frac{\partial A_{y}}{\partial x}\right]^{\mathrm{T}}, \\
& \frac{\partial E_{x}}{\partial t}=-\int_{\mathbb{R}^{4}} p f \mathrm{~d} p \mathrm{~d} \mathbf{s}, \\
& \frac{\partial E_{y}}{\partial t}=-\frac{\partial^{2} A_{y}}{\partial x^{2}}+A_{y} \int_{\mathbb{R}^{4}} f \mathrm{~d} p \mathrm{~d} \mathbf{s}+\mathfrak{h} \int_{\mathbb{R}^{4}} s_{z} \frac{\partial f}{\partial x} \mathrm{~d} p \mathrm{~d} \mathbf{s}, \\
& \frac{\partial E_{z}}{\partial t}=-\frac{\partial^{2} A_{z}}{\partial x^{2}}+A_{z} \int_{\mathbb{R}^{4}} f \mathrm{~d} p \mathrm{~d} \mathbf{s}-\mathfrak{h} \int_{\mathbb{R}^{4}} s_{y} \frac{\partial f}{\partial x} \mathrm{~d} p \mathrm{~d} \mathbf{s}, \\
& \frac{\partial \mathbf{A}_{\perp}}{\partial t}=-\mathbf{E}_{\perp} \\
& \frac{\partial E_{x}}{\partial x}=\int_{\mathbb{R}^{4}} f \mathrm{~d} p \mathrm{~d} \mathbf{s}-1 .
\end{aligned}
$$

This reduced spin Vlasov-Maxwell system also possesses a noncanonical Poisson structure. For any two functionals $\mathcal{F}$ and $\mathcal{G}$ depending on the unknowns $f, \mathbf{E}$, and $\mathbf{A}_{\perp}$, the 
Poisson bracket reads

$$
\begin{aligned}
& \{\mathcal{F}, \mathcal{G}\}=\int_{\mathbb{R}^{5}} f\left[\frac{\delta \mathcal{F}}{\delta f}, \frac{\delta \mathcal{G}}{\delta f}\right]_{x p} \mathrm{~d} x \mathrm{~d} p \mathrm{~d} \mathbf{s}+\int_{\mathbb{R}^{5}}\left(\frac{\delta \mathcal{F}}{\delta E_{x}} \frac{\partial f}{\partial p} \frac{\delta \mathcal{G}}{\delta f}-\frac{\delta \mathcal{G}}{\delta E_{x}} \frac{\partial f}{\partial p} \frac{\delta \mathcal{F}}{\delta f}\right) \mathrm{d} x \mathrm{~d} p \mathrm{~d} \mathbf{s} \\
& +\int_{\mathbb{R}}\left(\frac{\delta \mathcal{G}}{\delta \mathbf{A}_{\perp}} \cdot \frac{\delta \mathcal{F}}{\delta \mathbf{E}_{\perp}}-\frac{\delta \mathcal{F}}{\delta \mathbf{A}_{\perp}} \cdot \frac{\delta \mathcal{G}}{\delta \mathbf{E}_{\perp}}\right) \mathrm{d} x+\frac{1}{\mathfrak{h}} \int_{\mathbb{R}^{5}} f \mathbf{s} \cdot\left(\frac{\partial}{\partial \mathbf{s}} \frac{\delta \mathcal{F}}{\delta f} \times \frac{\partial}{\partial \mathbf{s}} \frac{\delta \mathcal{G}}{\delta f}\right) \mathrm{d} x \mathrm{~d} p \mathrm{~d} \mathbf{s}
\end{aligned}
$$

whereas the Hamiltonian functional, composed of the sum of kinetic, electric, magnetic and Zeeman (spin-dependent) energies, is defined by

$$
\begin{aligned}
\mathcal{H}\left(f, \mathbf{E}, \mathbf{A}_{\perp}\right) & =\underbrace{\frac{1}{2} \int_{\mathbb{R}^{5}} p^{2} f \mathrm{~d} x \mathrm{~d} p \mathrm{~d} \mathbf{s}+\frac{1}{2} \int_{\mathbb{R}^{5}}\left|\mathbf{A}_{\perp}\right|^{2} f \mathrm{~d} x \mathrm{~d} p \mathrm{~d} \mathbf{s}}_{\text {kinetic energy }}+\underbrace{\frac{1}{2} \int_{\mathbb{R}}|\mathbf{E}|^{2} \mathrm{~d} x}_{\text {electric energy }} \\
& +\underbrace{\frac{1}{2} \int_{\mathbb{R}}\left|\frac{\partial \mathbf{A}_{\perp}}{\partial x}\right|^{2} \mathrm{~d} x}_{\text {magnetic energy }}+\underbrace{\mathfrak{h} \int_{\mathbb{R}^{5}}\left(s_{y} \frac{\partial A_{z}}{\partial x}-s_{z} \frac{\partial A_{y}}{\partial x}\right) f \mathrm{~d} x \mathrm{~d} p \mathrm{~d} \mathbf{s}}_{\text {Zeeman energy }} .
\end{aligned}
$$

Thus, the reduced spin Vlasov-Maxwell system of equations 2.7 can be reformulated as

$$
\frac{\partial \mathcal{Z}}{\partial t}=\{\mathcal{Z}, \mathcal{H}\}
$$

where $\mathcal{Z}=\left(f, E_{x}, E_{y}, E_{z}, A_{y}, A_{z}\right)$ denotes the unknowns of the system, $\{\cdot, \cdot\}$ is defined by (2.8), and the Hamiltonian $\mathcal{H}$ is given by (2.9). In this work, periodic boundary conditions will be considered in the $x$ and $\mathbf{s}$ directions $\left(x \in[0, L], L>0, \mathbf{s} \in \mathbb{R}^{3}\right)$ and vanishing boundary condition in $p \in \mathbb{R}$. An initial condition $\mathcal{Z}(t=0)=\mathcal{Z}_{0}=\left(f_{0}, \mathbf{E}_{0}, \mathbf{A}_{\perp, 0}\right)$ supplements the system. The Poisson bracket (2.8) can be derived from (2.4) using chain rules of functional derivatives based on a similar change of unknowns proposed in Li et al. $(2019 b)$.

\section{GEMPIC formalism for the reduced spin Vlasov-Maxwell system}

In the framework introduced in Kraus et al. (2017), the Vlasov-Maxwell equations are discretized through a standard particle-in-cell ansatz for the distribution function and compatible finite elements for the electromagnetic fields. Then, the semi-discretization is obtained by inserting the ansatz into the Hamiltonian 2.9 and the Poisson bracket (2.8) of the reduced spin Vlasov-Maxwell equations. In this section, we will apply and detail the strategy for our spin Vlasov-Maxwell system (2.7).

Following Kraus et al. (2017), we consider the components of the electromagnetic fields separately and we consider $E_{x}, B_{y}, B_{z}$ as 1 -forms and $E_{y}, E_{z}, A_{y}, A_{z}$ as 0 -forms. 0 -forms and 1-forms are discretized in finite element spaces $V_{0} \subset H^{1}$ and $V_{1} \subset L^{2}$ respectively ( $H^{1}$ denotes the Sobolev space). There exists a commuting diagram (see (3.1) for the involved functional spaces in one spatial dimension (with periodic boundary conditions), between continuous spaces in the upper line and discrete subspaces in the lower line. The projectors $\Pi_{0}$ and $\Pi_{1}$ must be constructed carefully in order to assure the diagram to be commuting (Kraus et al. 2017).

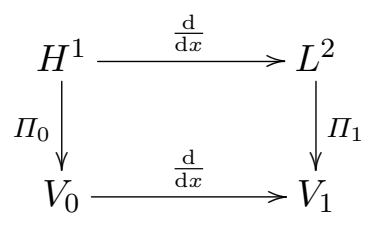


The basis for each of the finite dimensional spaces $V_{0}, V_{1}$, with $\operatorname{dim} V_{k}=N_{k}(\mathrm{k}=0$, 1 ) is denoted as: $\left\{\Lambda_{j}^{0}\right\}_{j=1, \cdots, N_{0}}$, and $\left\{\Lambda_{j}^{1}\right\}_{j=1, \cdots, N_{1}}$. The dual bases of $V_{0}$ and $V_{1}$ are $\left\{\Sigma_{j}^{0}\right\}_{j=1, \cdots, N_{0}}$, and $\left\{\Sigma_{j}^{1}\right\}_{j=1, \cdots, N_{1}}$ respectively, ie, $\int \Sigma_{i}^{k} \Lambda_{j}^{k} \mathrm{~d} x=\delta_{i, j}, k=0,1$.

In this paper, we choose B-splines as the basis functions, and $N_{0}=N_{1}=M$. The spatial domain $[0, L]$ is discretized by a uniform grid

$$
x_{j}=(j-1) \Delta x, \Delta x=L / M, j=1, \cdots, M .
$$

The B-splines basis functions $\left\{\Lambda_{j}^{0}\right\}_{j=1, \cdots, N_{0}}$, and $\left\{\Lambda_{j}^{1}\right\}_{j=1, \cdots, N_{1}}$ for $V_{0}=\operatorname{span}\left\{\left\{\Lambda_{j}^{0}\right\}_{j=1, \cdots, N_{0}}\right\}$, and $V_{1}=\operatorname{span}\left\{\left\{\Lambda_{j}^{1}\right\}_{j=1, \cdots, N_{1}}\right\}$ are given by

$$
\Lambda_{j}^{0}(x)=\frac{1}{6}\left\{\begin{array}{l}
\left(2-\frac{\left|x-x_{j+2}\right|}{\Delta x}\right)^{3}, \quad 1 \leqslant \frac{\left|x-x_{j+2}\right|}{\Delta x} \leqslant 2, \\
4-6\left(\frac{\left|x-x_{j+2}\right|}{\Delta x}\right)^{2}+3\left(\frac{\left|x-x_{j+2}\right|}{\Delta x}\right)^{3}, \quad 0 \leqslant \frac{\left|x-x_{j+2}\right|}{\Delta x} \leqslant 1, \\
0, \quad \text { otherwise, }
\end{array}\right.
$$

and

$$
\Lambda_{j}^{1}(x)=\frac{1}{2}\left\{\begin{array}{l}
\left(\frac{x-x_{j}}{\Delta x}\right)^{2}, \quad 0 \leqslant \frac{x-x_{j}}{\Delta x} \leqslant 1, \\
-2\left(\frac{x-x_{j}}{\Delta x}\right)^{2}+6\left(\frac{x-x_{j}}{\Delta x}\right)-3, \quad 1 \leqslant \frac{x-x_{j}}{\Delta x} \leqslant 2, \\
\left(3-\frac{x-x_{j}}{\Delta x}\right)^{2}, \quad 2 \leqslant \frac{x-x_{j}}{\Delta x} \leqslant 3, \\
0, \quad \text { otherwise. }
\end{array}\right.
$$

The important relation between $\Lambda^{1}$ and $\Lambda^{0}$

$$
\frac{\mathrm{d}}{\mathrm{d} x} \Lambda_{j}^{0}(x)=\frac{1}{\Delta x}\left(\Lambda_{j}^{1}(x)-\Lambda_{j+1}^{1}(x)\right)
$$

can be reformulated as

$$
\frac{\mathrm{d}}{\mathrm{d} x}\left(\Lambda_{1}^{0}, \cdots, \Lambda_{N_{0}}^{0}\right)(x)=\left(\Lambda_{1}^{1}, \cdots, \Lambda_{N_{1}}^{1}\right)(x) \mathbb{C},
$$

where $\mathbb{C}$ is a matrix of size $N_{1} \times N_{0}=M \times M$, given by

$$
\mathbb{C}=\frac{1}{\Delta x}\left(\begin{array}{ccccccc}
1 & 0 & 0 & 0 & \cdots & 0 & -1 \\
-1 & 1 & 0 & 0 & \cdots & 0 & 0 \\
0 & -1 & 1 & 0 & \cdots & 0 & 0 \\
0 & 0 & \ddots & \ddots & \ddots & \vdots & \vdots \\
\vdots & \vdots & \ddots & \ddots & \ddots & \ddots & \vdots \\
\vdots & \vdots & \vdots & \ddots & \ddots & \ddots & 0 \\
0 & 0 & 0 & \cdots & 0 & -1 & 1
\end{array}\right)
$$

Then, each element of the finite-dimensional spaces $V_{0}$ and $V_{1}$ can be expanded on their respective basis functions. For the electric field components $\left(E_{x}, E_{y}, E_{z}\right) \in L^{2} \times H^{1} \times H^{1}$, there are approximated by $\left(E_{x, h}, E_{y, h}, E_{z, h}\right) \in V_{1} \times V_{0} \times V_{0}$ according to the following 
representation

$E_{x, h}(t, x)=\sum_{j=1}^{N_{1}} e_{x, j}(t) \Lambda_{j}^{1}(x), E_{y, h}(t, x)=\sum_{j=1}^{N_{0}} e_{y, j}(t) \Lambda_{j}^{0}(x), E_{z, h}(t, x)=\sum_{j=1}^{N_{0}} e_{z, j}(t) \Lambda_{j}^{0}(x)$,

whereas for the vector potential components $\left(A_{y}, A_{z}\right) \in H^{1} \times H^{1}$, there are approximated by $\left(A_{y, h}, A_{z, h}\right) \in V_{0} \times V_{0}$ according to

$$
A_{y, h}(t, x)=\sum_{j=1}^{N_{0}} a_{y, j}(t) \Lambda_{j}^{0}(x), A_{z, h}(t, x)=\sum_{j=1}^{N_{0}} a_{z, j}(t) \Lambda_{j}^{0}(x)
$$

Regarding the distribution function $f(t, x, p, \mathbf{s})$, we use the following representation

$$
f(t, x, p, \mathbf{s}) \approx f_{h}(t, x, p, \mathbf{s})=\sum_{a=1}^{N_{p}} \omega_{a} \delta\left(x-x_{a}(t)\right) \delta\left(p-p_{a}(t)\right) \delta\left(\mathbf{s}-\mathbf{s}_{a}(t)\right),
$$

where $\omega_{a}, x_{a}, p_{a}$, and $\mathbf{s}_{a}$ denote respectively the weight, the position, the momentum (velocity), and the spin co-ordinates of $a$-th particle.

\subsection{Derivation of the discrete Poisson bracket}

Using the above approximation of the electromagnetic fields (3.6)-(3.7) and of the distribution function (3.8), we shall construct a discrete geometric structure (discrete Poisson bracket and discrete Hamiltonian) from which the equations of motion will be derived. Specifically, discrete functional derivatives are derived based on the discrete representation of the unknowns (3.6)-(3.7) (see Appendix A for more details), which are inserted into the continuous Poisson bracket (2.8) to derive the discrete Poisson bracket.

We introduce some notations to make the expressions simpler. First, we introduce the discrete time-dependent unknowns for the electromagnetic fields

$$
\begin{aligned}
& \mathbf{e}_{x}=\left(e_{x, 1}, \cdots, e_{x, N_{1}}\right)^{\mathrm{T}} \in \mathbb{R}^{N_{1}}, \mathbf{e}_{y}=\left(e_{y, 1}, \cdots, e_{y, N_{0}}\right)^{\mathrm{T}} \in \mathbb{R}^{N_{0}}, \mathbf{e}_{z}=\left(e_{z, 1}, \cdots, e_{z, N_{0}}\right)^{\mathrm{T}} \in \mathbb{R}^{N_{0}}, \\
& \mathbf{a}_{y}=\left(a_{y, 1}, \cdots, a_{y, N_{0}}\right)^{\mathrm{T}} \in \mathbb{R}^{N_{0}}, \mathbf{a}_{z}=\left(a_{z, 1}, \cdots, a_{z, N_{0}}\right)^{\mathrm{T}} \in \mathbb{R}^{N_{0}},
\end{aligned}
$$

and for the particles (position, velocity and spin)

$$
\begin{aligned}
& \mathbf{X}=\left(x_{1}, \cdots, x_{N_{p}}\right)^{\mathrm{T}} \in \mathbb{R}^{N_{p}}, \mathbf{P}=\left(p_{1}, \cdots, p_{N_{p}}\right)^{\mathrm{T}} \in \mathbb{R}^{N_{p}}, \\
& \mathbf{S}_{i}=\left(s_{1, i}, \cdots, s_{a, i}, \cdots, s_{N_{p}, i}\right)^{\mathrm{T}} \in \mathbb{R}^{N_{p}}, i \in\{x, y, z\}, \\
& \mathbf{S}=\left(\mathbf{s}_{1}, \mathbf{s}_{2}, \cdots, \mathbf{s}_{a}, \cdots, \mathbf{s}_{N_{p}}\right)^{\mathrm{T}} \in \mathbb{R}^{3 N_{p}} \text { with } \mathbf{s}_{a}=\left(s_{a, x}, s_{a, y}, s_{a, z}\right) \in \mathbb{R}^{3} .
\end{aligned}
$$

Moreover, we will need some matrix notations to take into account the fields-particles 
coupling:

$$
\begin{aligned}
& \Lambda_{k}(\mathbf{X})=\left(\begin{array}{ccc}
\Lambda_{1}^{k}\left(x_{1}\right) & \cdots & \Lambda_{N_{k}}^{k}\left(x_{1}\right) \\
\Lambda_{1}^{k}\left(x_{2}\right) & \cdots & \Lambda_{N_{k}}^{k}\left(x_{2}\right) \\
\vdots & \ddots & \vdots \\
\Lambda_{1}^{k}\left(x_{N_{p}}\right) & \cdots & \Lambda_{N_{k}}^{k}\left(x_{N_{p}}\right)
\end{array}\right) \in \mathcal{M}_{N_{p}, N_{k}}(\mathbb{R}), \quad \text { for } k=0,1, \\
& \mathbb{M}_{k}=\left(\begin{array}{ccc}
\int \Lambda_{1}^{k}(x) \Lambda_{1}^{k}(x) \mathrm{d} x & \cdots & \int \Lambda_{1}^{k}(x) \Lambda_{N_{k}}^{k}(x) \mathrm{d} x \\
\int \Lambda_{2}^{k}(x) \Lambda_{1}^{k}(x) \mathrm{d} x & \cdots & \int \Lambda_{2}^{k}(x) \Lambda_{N_{k}}^{k}(x) \mathrm{d} x \\
\vdots & \ddots & \vdots \\
\int \Lambda_{N_{k}}^{k}(x) \Lambda_{1}^{k}(x) \mathrm{d} x & \cdots & \int \Lambda_{N_{k}}^{k}(x) \Lambda_{N_{k}}^{k}(x) \mathrm{d} x
\end{array}\right) \in \mathcal{M}_{N_{k}, N_{k}}(\mathbb{R}), \quad \text { for } k=0,1, \\
& \mathbb{N}_{k}\left(x_{a}\right)=\left(\begin{array}{ccc}
\Lambda_{1}^{k}\left(x_{a}\right) \Lambda_{1}^{k}\left(x_{a}\right) & \cdots & \Lambda_{1}^{k}\left(x_{a}\right) \Lambda_{N_{k}}^{k}\left(x_{a}\right) \\
\Lambda_{2}^{k}\left(x_{a}\right) \Lambda_{1}^{k}\left(x_{a}\right) & \cdots & \Lambda_{2}^{k}\left(x_{a}\right) \Lambda_{N_{k}}^{k}\left(x_{a}\right) \\
\vdots & \ddots & \vdots \\
\Lambda_{N_{k}}^{k}\left(x_{a}\right) \Lambda_{1}^{k}\left(x_{a}\right) & \cdots & \Lambda_{N_{k}}^{k}\left(x_{a}\right) \Lambda_{N_{k}}^{k}\left(x_{a}\right)
\end{array}\right) \in \mathcal{M}_{N_{k}, N_{k}(\mathbb{R}), \quad \text { for } k=0,1,}, \\
& \Lambda^{k}\left(x_{a}\right)=\left(\Lambda_{1}^{k}\left(x_{a}\right), \cdots, \Lambda_{N_{k}}^{k}\left(x_{a}\right)\right)^{\mathrm{T}} \in \mathbb{R}^{N_{k}}, \quad \text { for } k=0,1 .
\end{aligned}
$$

Finally, we introduce the weight matrix and some matrices related to the spin particles

$$
\begin{aligned}
& \mathbb{W}=\operatorname{diag}\left(\omega_{1}, \cdots, \omega_{N_{p}}\right) \in \mathcal{M}_{N_{p}, N_{p}}(\mathbb{R}), \\
& \mathbb{S}_{a}=\frac{1}{\omega_{a}}\left(\begin{array}{ccc}
0 & s_{a, z} & -s_{a, y} \\
-s_{a, z} & 0 & s_{a, x} \\
s_{a, y} & -s_{a, x} & 0
\end{array}\right) \in \mathcal{M}_{3,3}(\mathbb{R}), \\
& \mathbb{S}=\operatorname{diag}\left(\mathbb{S}_{1}, \cdots, \mathbb{S}_{N_{p}}\right) \in \mathcal{M}_{3 N_{p}, 3 N_{p}}(\mathbb{R}) .
\end{aligned}
$$

Any functional $\mathcal{F}$ of a continuous representation of the approximated fields or distribution function $\left(f_{h}, E_{x, h}, E_{y, h}, E_{z, h}, A_{y, h}, A_{z, h}\right)$ is now considered as a new function $F$ of the discrete unknowns (particles unknowns $\mathbf{X}, \mathbf{P}, \mathbf{S}$, and fields finite-element coefficients $\left.\mathbf{e}_{x}, \mathbf{e}_{y}, \mathbf{e}_{z}, \mathbf{a}_{y}, \mathbf{a}_{z}\right)$, ie:

$$
F(\mathbf{u}):=F\left(\mathbf{X}, \mathbf{P}, \mathbf{S}, \mathbf{e}_{x}, \mathbf{e}_{y}, \mathbf{e}_{z}, \mathbf{a}_{y}, \mathbf{a}_{z}\right):=\mathcal{F}\left[f_{h}, E_{x, h}, E_{y, h}, E_{z, h}, A_{y, h}, A_{z, h}\right] .
$$

This representation enables us to replace all functional derivatives in (2.8) by their discrete counterparts (see Appendix Afor more details). We then obtain the semi-discrete Poisson bracket

$$
\begin{aligned}
\{F, G\}(\mathbf{u}) & =\{F, G\}\left(\mathbf{X}, \mathbf{P}, \mathbf{S}, \mathbf{e}_{x}, \mathbf{e}_{y}, \mathbf{e}_{z}, \mathbf{a}_{y}, \mathbf{a}_{z}\right) \\
& =\sum_{a=1}^{N_{p}} \frac{1}{\omega_{a}}\left(\frac{\partial F}{\partial x_{a}} \frac{\partial G}{\partial p_{a}}-\frac{\partial G}{\partial x_{a}} \frac{\partial F}{\partial p_{a}}\right)+\frac{1}{\mathfrak{h}} \sum_{a=1}^{N_{p}}\left(\mathbb{S}_{a} \frac{\partial F}{\partial \mathbf{s}_{a}}\right) \cdot \frac{\partial G}{\partial \mathbf{s}_{a}} \\
& +\sum_{a=1}^{N_{p}} \sum_{i, j=1}^{N_{1}}\left(\frac{\partial F}{\partial e_{x, i}}\left(\mathbb{M}_{1}^{-1}\right)_{i j} \Lambda_{j}^{1}\left(x_{a}\right) \frac{\partial G}{\partial p_{a}}\right)-\sum_{a=1}^{N_{p}} \sum_{i, j=1}^{N_{1}}\left(\frac{\partial G}{\partial e_{x, i}}\left(\mathbb{M}_{1}^{-1}\right)_{i j} \Lambda_{j}^{1}\left(x_{a}\right) \frac{\partial F}{\partial p_{a}}\right) \\
& +\left(\frac{\partial G}{\partial \mathbf{a}_{y}}\right)^{\mathrm{T}} \mathbb{M}_{0}^{-1} \frac{\partial F}{\partial \mathbf{e}_{y}}+\left(\frac{\partial G}{\partial \mathbf{a}_{z}}\right)^{\mathrm{T}} \mathbb{M}_{0}^{-1} \frac{\partial F}{\partial \mathbf{e}_{z}}-\left(\frac{\partial F}{\partial \mathbf{a}_{y}}\right)^{\mathrm{T}} \mathbb{M}_{0}^{-1} \frac{\partial G}{\partial \mathbf{e}_{y}}-\left(\frac{\partial F}{\partial \mathbf{a}_{z}}\right)^{\mathrm{T}} \mathbb{M}_{0}^{-1} \frac{\partial G}{\partial \mathbf{e}_{z}} .
\end{aligned}
$$

By rearranging the terms, we are able to obtain the following finite-dimensional Poisson bracket

$$
\{F, G\}=\left(\nabla_{\mathbf{u}} F\right)^{\mathrm{T}} \mathbb{J}(\mathbf{u}) \nabla_{\mathbf{u}} G
$$


Geometric PIC for the Vlasov-Maxwell equations with spin effects

where $\mathbf{u}=\left(\mathbf{X}, \mathbf{P}, \mathbf{S}, \mathbf{e}_{x}, \mathbf{e}_{y}, \mathbf{e}_{z}, \mathbf{a}_{y}, \mathbf{a}_{z}\right)^{\mathrm{T}}$ and the matrix $\mathbb{l}(\mathbf{u})$ is defined by

$$
\rrbracket(\mathbf{u})=\left(\begin{array}{cccccccc}
\mathbf{0} & \mathbb{W}^{-1} & \mathbf{0} & \mathbf{0} & \mathbf{0} & \mathbf{0} & \mathbf{0} & \mathbf{0} \\
-W^{-1} & \mathbf{0} & \mathbf{0} & \Lambda_{1}(\mathbf{X}) M_{1}^{-1} & \mathbf{0} & \mathbf{0} & \mathbf{0} & \mathbf{0} \\
\mathbf{0} & \mathbf{0} & \frac{1}{\mathfrak{h}} S & \mathbf{0} & \mathbf{0} & \mathbf{0} & \mathbf{0} & \mathbf{0} \\
\mathbf{0} & -M_{1}^{-1} \Lambda_{1}(\mathbf{X})^{\mathrm{T}} & \mathbf{0} & \mathbf{0} & \mathbf{0} & \mathbf{0} & \mathbf{0} & \mathbf{0} \\
\mathbf{0} & \mathbf{0} & \mathbf{0} & \mathbf{0} & \mathbf{0} & \mathbf{0} & M_{0}^{-1} & \mathbf{0} \\
\mathbf{0} & \mathbf{0} & \mathbf{0} & \mathbf{0} & \mathbf{0} & \mathbf{0} & \mathbf{0} & \mathbb{M}_{0}^{-1} \\
\mathbf{0} & \mathbf{0} & \mathbf{0} & \mathbf{0} & -M_{0}^{-1} & \mathbf{0} & \mathbf{0} & \mathbf{0} \\
\mathbf{0} & \mathbf{0} & \mathbf{0} & \mathbf{0} & \mathbf{0} & -M_{0}^{-1} & \mathbf{0} & \mathbf{0}
\end{array}\right)
$$

The following Theorem states that the bracket defined by $(3.13)-(3.14)$ is indeed a Poisson bracket.

THEOREM 1. The above bracket (3.13)-(3.14) is a Poisson bracket.

Proof. See Appendix B.

\subsection{Discrete Hamiltonian and equations of motion}

The discrete Hamiltonian is obtained by inserting the representation of the unknowns (3.6), (3.7) and (3.8) into the Hamiltonian (2.9). With $\mathbf{u}=\left(\mathbf{X}, \mathbf{P}, \mathbf{S}, \mathbf{e}_{x}, \mathbf{e}_{y}, \mathbf{e}_{z}, \mathbf{a}_{y}, \mathbf{a}_{z}\right)^{\mathrm{T}}$, we have

$$
\begin{aligned}
H(\mathbf{u}) & =\frac{1}{2} \int p^{2} \sum_{a=1}^{N_{p}} \omega_{a} \delta\left(x-x_{a}\right) \delta\left(p-p_{a}\right) \delta\left(\mathbf{s}-\mathbf{s}_{a}\right) \mathrm{d} x \mathrm{~d} p \mathrm{~d} \mathbf{s} \\
& +\frac{1}{2} \int \sum_{a=1}^{N_{p}}\left(\left|\sum_{j=1}^{N_{0}} a_{y, j} \Lambda_{j}^{0}(x)\right|^{2}+\left|\sum_{j=1}^{N_{0}} a_{z, j} \Lambda_{j}^{0}(x)\right|^{2}\right) \omega_{a} \delta\left(x-x_{a}\right) \delta\left(p-p_{a}\right) \delta\left(\mathbf{s}-\mathbf{s}_{a}\right) \mathrm{d} x \mathrm{~d} p \mathrm{~d} \mathbf{s} \\
& +\frac{1}{2} \int\left|\sum_{j=1}^{N_{1}} e_{x, j} \Lambda_{j}^{1}(x)\right|^{2} \mathrm{~d} x+\frac{1}{2} \int\left(\left|\sum_{j=1}^{N_{0}} e_{y, j} \Lambda_{j}^{0}(x)\right|^{2}+\left|\sum_{j=1}^{N_{0}} e_{z, j} \Lambda_{j}^{0}(x)\right|^{2}\right) \mathrm{d} x \\
& +\frac{1}{2} \int\left|\frac{\mathrm{d}}{\mathrm{d} x} \sum_{a=1}^{N_{0}} a_{y, j} \Lambda_{j}^{0}(x)\right|^{2} \mathrm{~d} x+\frac{1}{2} \int\left|\frac{\mathrm{d}}{\mathrm{d} x} \sum_{a=1}^{N_{0}} a_{z, j} \Lambda_{j}^{0}(x)\right|^{2} \mathrm{~d} x \\
& +\mathfrak{h} \int \sum_{a=1}^{N_{p}}\left(s_{y} \frac{\mathrm{d}}{\mathrm{d} x} \sum_{j=1}^{N_{0}} a_{z, j} \Lambda_{j}^{0}(x)-s_{z} \frac{\mathrm{d}}{\mathrm{d} x} \sum_{j=1}^{N_{0}} a_{y, j} \Lambda_{j}^{0}(x)\right) \omega_{a} \delta\left(x-x_{a}\right) \delta\left(p-p_{a}\right) \delta\left(\mathbf{s}-\mathbf{s}_{a}\right) \mathrm{d} x \mathrm{~d} p \mathrm{~d} \mathbf{s} .
\end{aligned}
$$

Using the notations $(3.9),(3.10)$ and $(3.11)$ introduced in the preceding section, the above formula can be written more compactly as

$$
\begin{aligned}
H(\mathbf{u}) & =\frac{1}{2} \mathbf{P}^{\mathrm{T}} \mathbb{W} \mathbf{P}+\frac{1}{2} \sum_{a=1}^{N_{p}} \omega_{a} \mathbf{a}_{y}^{\mathrm{T}} \mathbb{N}_{0}\left(x_{a}\right) \mathbf{a}_{y}+\frac{1}{2} \sum_{a=1}^{N_{p}} \omega_{a} \mathbf{a}_{z}^{\mathrm{T}} \mathbb{N}_{0}\left(x_{a}\right) \mathbf{a}_{z} \\
& +\frac{1}{2} \mathbf{e}_{x}^{\mathrm{T}} \mathrm{M}_{1} \mathbf{e}_{x}+\frac{1}{2} \mathbf{e}_{y}^{\mathrm{T}} \mathrm{M}_{0} \mathbf{e}_{y}+\frac{1}{2} \mathbf{e}_{z}^{\mathrm{T}} \mathrm{M}_{0} \mathbf{e}_{z}+\frac{1}{2} \mathbf{a}_{y}^{\mathrm{T}} \mathbb{C}^{\mathrm{T}} \mathbb{M}_{1} \mathbb{C} \mathbf{a}_{y}+\frac{1}{2} \mathbf{a}_{z}^{\mathrm{T}} \mathbb{C}^{\mathrm{T}} \mathbb{M}_{1} \mathbb{C} \mathbf{a}_{z} \\
& +\mathfrak{h} \mathbf{a}_{z}^{\mathrm{T}} \mathbb{C}^{\mathrm{T}} \Lambda_{1}(\mathbf{X})^{\mathrm{T}} \mathfrak{W} \mathbf{S}_{y}-\mathfrak{h} \mathbf{a}_{y}^{\mathrm{T}} \mathbb{C}^{\mathrm{T}} \Lambda_{1}(\mathbf{X})^{\mathrm{T}} \mathfrak{W} \mathbf{S}_{z} .
\end{aligned}
$$


From the discrete Poisson bracket (3.13)- 3.14 and the discrete Hamiltonian (3.15), the equations of motion then read as

$$
\dot{\mathbf{u}}=\{\mathbf{u}, H\}=\mathbb{J}(\mathbf{u}) \nabla_{\mathbf{u}} H, \quad \mathbf{u}(t=0)=\mathbf{u}_{0}
$$

Specifically, we obtain the following equations of motion

$$
\begin{aligned}
& \dot{\mathbf{X}}=\mathbf{P}, \quad \dot{\mathbf{P}}=-\mathbb{W}^{-1} \frac{\partial H}{\partial \mathbf{X}}+\Lambda_{1}(\mathbf{X}) \mathbf{e}_{x}, \quad \dot{\mathbf{S}}=\frac{1}{\mathfrak{h}} \mathbb{S} \frac{\partial H}{\partial \mathbf{S}}, \\
& \dot{\mathbf{e}}_{x}=-\mathbb{M}_{1}^{-1} \Lambda_{1}(\mathbf{X})^{\mathrm{T}} \mathbb{W} \mathbf{P}, \\
& \dot{\mathbf{e}}_{y}=-\mathbb{M}_{0}^{-1} \mathbb{C}^{\mathrm{T}} \Lambda_{1}(\mathbf{X})^{\mathrm{T}} \mathbb{W} \mathbf{S}_{z}+\mathbb{M}_{0}^{-1}\left(\sum_{a=1}^{N_{p}} \omega_{a} \mathbb{N}_{0}\left(x_{a}\right) \mathbf{a}_{y}+\mathbb{C}^{\mathrm{T}} \mathbb{M}_{1} \mathbb{C} \mathbf{a}_{y}\right), \\
& \dot{\mathbf{e}}_{z}=\mathbb{M}_{0}^{-1} \mathbb{C}^{\mathrm{T}} \Lambda_{1}(\mathbf{X})^{\mathrm{T}} \mathbb{W} \mathbf{S}_{y}+\mathbb{M}_{0}^{-1}\left(\sum_{a=1}^{N_{p}} \omega_{a} \mathbb{N}_{0}\left(x_{a}\right) \mathbf{a}_{z}+\mathbb{C}^{\mathrm{T}} \mathbb{M}_{1} \mathbb{C} \mathbf{a}_{z}\right), \\
& \dot{\mathbf{a}}_{y}=-\mathbf{e}_{y}, \quad \dot{\mathbf{a}}_{z}=-\mathbf{e}_{z},
\end{aligned}
$$

where

$$
\begin{aligned}
& \frac{\partial H}{\partial x_{a}}=\frac{w_{a}}{2} \mathbf{a}_{y}^{\mathrm{T}} \mathbb{L} \mathbf{a}_{y}+\frac{w_{a}}{2} \mathbf{a}_{z}^{\mathrm{T}} \mathbb{L} \mathbf{a}_{z}+\mathfrak{h} \mathbf{a}_{z}^{\mathrm{T}} \mathbb{C}^{\mathrm{T}} \frac{\partial\left(\Lambda_{1}(\mathbf{X})\right)^{\mathrm{T}}}{\partial x_{a}} \mathfrak{W} \mathbf{S}_{y}-\mathfrak{h} \mathbf{a}_{y}^{\mathrm{T}} \mathbb{C}^{\mathrm{T}} \frac{\partial\left(\Lambda_{1}(\mathbf{X})\right)^{\mathrm{T}}}{\partial x_{a}} \mathbb{W}_{z}, \\
& \text { with } \mathbb{L}=\left(\frac{\partial}{\partial x_{a}} \Lambda^{0}\left(x_{a}\right) \Lambda^{0}\left(x_{a}\right)^{\mathrm{T}}+\Lambda^{0}\left(x_{a}\right) \frac{\partial}{\partial x_{a}} \Lambda^{0}\left(x_{a}\right)^{\mathrm{T}}\right),
\end{aligned}
$$

and finally for $\frac{\partial H}{\partial \mathbf{S}}$ (recalling $\mathbf{S}=\left(\mathbf{s}_{1}, \mathbf{s}_{2}, \cdots, \mathbf{s}_{a}, \cdots, \mathbf{s}_{N_{p}}\right)^{\mathrm{T}} \in \mathbb{R}^{3 N_{p}}$ ), we get for the spin variables of $a$-th particle,

$$
\frac{\partial H}{\partial s_{a, x}}=0, \quad \frac{\partial H}{\partial s_{a, y}}=\mathfrak{h} \mathbf{a}_{z}^{\mathrm{T}} \mathbb{C}^{\mathrm{T}} \Lambda^{1}\left(x_{a}\right) \omega_{a}, \quad \frac{\partial H}{\partial s_{a, z}}=-\mathfrak{h} \mathbf{a}_{y}^{\mathrm{T}} \mathbb{C}^{\mathrm{T}} \Lambda^{1}\left(x_{a}\right) \omega_{a}
$$

\section{Hamiltonian splitting method}

Once the semi-discretization is performed, the resulting Poisson system has to be integrated in time. Here a Hamiltonian splitting method (Crouseilles et al. 2015, Li et al. $2019 a$ ) is adopted, in which the solution is obtained as compositions of exact solutions of Hamiltonian subsystems. Hence, such resulting schemes are Poisson integrators in the sense of Hairer et al. (2002). Moreover, as we will see, each substep is explicit in time, and can be used to derive higher-order methods, taking into account some specific commutator relations (Casas et al. 2017; Hairer et al. 2002). By splitting the discrete Hamiltonian $H$ given by $(3.15)$ into the following four parts

$$
H=H_{p}+H_{A}+H_{s}+H_{E},
$$


where

$$
\begin{aligned}
H_{p} & =\frac{1}{2} \mathbf{P}^{\mathrm{T}} \boldsymbol{W} \mathbf{P} \\
H_{A} & =\frac{1}{2} \sum_{a=1}^{N_{p}} \omega_{a} \mathbf{a}_{y}^{\mathrm{T}} \mathbb{N}_{0}\left(x_{a}\right) \mathbf{a}_{y}+\frac{1}{2} \sum_{a=1}^{N_{p}} \omega_{a} \mathbf{a}_{z}^{\mathrm{T}} \mathbb{N}_{0}\left(x_{a}\right) \mathbf{a}_{z}+\frac{1}{2} \mathbf{a}_{y}^{\mathrm{T}} \mathbb{C}^{\mathrm{T}} \mathbb{M}_{1} \mathbb{C} \mathbf{a}_{y}+\frac{1}{2} \mathbf{a}_{z}^{\mathrm{T}} \mathbb{C}^{\mathrm{T}} \mathbb{M}_{1} \mathbb{C} \mathbf{a}_{z}, \\
H_{s} & =\mathfrak{h} \mathbf{a}_{z}^{\mathrm{T}} \mathbb{C}^{\mathrm{T}} \Lambda_{1}(\mathbf{X})^{\mathrm{T}} \mathfrak{W} \mathbf{S}_{y}-\mathfrak{h} \mathbf{a}_{y}^{\mathrm{T}} \mathbb{C}^{\mathrm{T}} \Lambda_{1}(\mathbf{X})^{\mathrm{T}} \mathfrak{W} \mathbf{S}_{z}, \\
H_{E} & =\frac{1}{2} \mathbf{e}_{x}^{\mathrm{T}} \mathbb{M}_{1} \mathbf{e}_{x}+\frac{1}{2} \mathbf{e}_{y}^{\mathrm{T}} \mathbb{M}_{0} \mathbf{e}_{y}+\frac{1}{2} \mathbf{e}_{z}^{\mathrm{T}} \mathbb{M}_{0} \mathbf{e}_{z},
\end{aligned}
$$

we are led to solve the four corresponding Hamiltonian subsystems

$$
\dot{\mathbf{u}}=\left\{\mathbf{u}, H_{p}\right\}, \dot{\mathbf{u}}=\left\{\mathbf{u}, H_{A}\right\}, \dot{\mathbf{u}}=\left\{\mathbf{u}, H_{s}\right\}, \dot{\mathbf{u}}=\left\{\mathbf{u}, H_{E}\right\},
$$

where $\mathbf{u}=\left(\mathbf{X}, \mathbf{P}, \mathbf{S}, \mathbf{e}_{x}, \mathbf{e}_{y}, \mathbf{e}_{z}, \mathbf{a}_{y}, \mathbf{a}_{z}\right)^{\mathrm{T}}$, and the corresponding solution flows are denoted by $\varphi_{t}^{\left[H_{p}\right]}, \varphi_{t}^{\left[H_{A}\right]}, \varphi_{t}^{\left[H_{s}\right]}$ and $\varphi_{t}^{\left[H_{E}\right]}$. Then, numerical solutions of 3.16 can be obtained, for instance, as a first order Lie splitting or a second order Strang splitting:

$$
\varphi_{t}^{\left[H_{p}\right]} \circ \varphi_{t}^{\left[H_{A}\right]} \circ \varphi_{t}^{\left[H_{s}\right]} \circ \varphi_{t}^{\left[H_{E}\right]}, \quad \varphi_{t / 2}^{\left[H_{p}\right]} \circ \varphi_{t / 2}^{\left[H_{A}\right]} \circ \varphi_{t / 2}^{\left[H_{s}\right]} \circ \varphi_{t}^{\left[H_{E}\right]} \circ \varphi_{t / 2}^{\left[H_{s}\right]} \circ \varphi_{t / 2}^{\left[H_{A}\right]} \circ \varphi_{t / 2}^{\left[H_{p}\right]} .
$$

Let us also remark that higher-order splitting schemes can be easily constructed by a suitable composition of the subflows $\varphi_{t}^{\left[H_{*}\right]}$ (see (Casas et al. 2017, Hairer et al. 2002)). It is worth mentioning that each subflow $\varphi_{t}^{\left[H_{*}\right]}$ can be solved exactly, which will be detailed in the following subsections.

\subsection{Subsystem $H_{p}$}

The subsystem corresponding to $H_{p}=\frac{1}{2} \mathbf{P}^{\mathrm{T}} \mathfrak{W} \mathbf{P}$ is $\dot{\mathbf{u}}=\mathbb{J}(\mathbf{u}) \nabla_{\mathbf{u}} H_{p}$, or specifically

$$
\begin{aligned}
& \dot{\mathbf{X}}=\mathbf{P}, \dot{\mathbf{P}}=\mathbf{0}, \dot{\mathbf{S}}=\mathbf{0}, \\
& \dot{\mathbf{e}}_{x}=-\mathbb{M}_{1}^{-1} \Lambda_{1}(\mathbf{X})^{\mathrm{T}} \mathbb{W} \mathbf{P}, \dot{\mathbf{e}}_{y}=\mathbf{0}, \dot{\mathbf{e}}_{z}=\mathbf{0}, \\
& \dot{\mathbf{a}}_{y}=\mathbf{0}, \dot{\mathbf{a}}_{z}=\mathbf{0} .
\end{aligned}
$$

For this subsystem, we only need to compute $\mathbf{X}, \mathbf{e}_{x}$ at time $t$

$$
\mathbf{X}(t)=\mathbf{X}(0)+t \mathbf{P}(0), \mathbb{M}_{1} \mathbf{e}_{x}(t)=\mathbb{M}_{1} \mathbf{e}_{x}(0)-\int_{0}^{t} \Lambda_{1}(\mathbf{X}(\tau))^{\mathrm{T}} \mathbb{W} \mathbf{P}(0) \mathrm{d} \tau
$$

REMARK 1. Multiplying $\mathbb{C}^{\mathrm{T}} \mathbb{M}_{1}$ from left with $\dot{\mathbf{e}}_{x}=-\mathbb{M}_{1}^{-1} \Lambda_{1}(\mathbf{X})^{\mathrm{T}} \mathbb{W} \mathbf{P}$, we get

$$
\mathbb{C}^{\mathrm{T}} \mathbf{M}_{1} \dot{\mathbf{e}}_{x}(t)=-\mathbb{C}^{\mathrm{T}} \Lambda_{1}(\mathbf{X})^{\mathrm{T}} \mathbb{W} \mathbf{P}
$$

As $\Lambda_{1}(\mathbf{X}) \mathbb{C}=\partial_{x} \Lambda_{0}(\mathbf{X})$, we get $\mathbb{C}^{\mathrm{T}} \mathbb{M}_{1} \dot{\mathbf{e}}_{x}(t)=-\partial_{x} \Lambda_{0}(\mathbf{X})^{\mathrm{T}} \mathbb{W} \mathbf{P}$ and using $\dot{\mathbf{X}}=\mathbf{P}$, we have

$$
\mathbb{C}^{\mathrm{T}} \mathrm{M}_{1} \dot{\mathbf{e}}_{x}(t)=-\frac{\mathrm{d} \Lambda_{0}(\mathbf{X})^{\mathrm{T}}}{\mathrm{d} t} \mathbb{W} \mathbb{1}_{N_{p}}
$$

with $\mathbb{1}_{N_{p}}$ the vector of size $N_{p}$ composed of 1 . Then, the discrete Poisson equation $\mathbb{C}^{\mathrm{T}} \mathrm{M}_{1} \mathbf{e}_{x}(t)=-\Lambda_{0}(\mathbf{X})^{\mathrm{T}} \mathrm{W} \mathbb{1}_{N_{p}}$ is always satisfied if it holds initially. 


\subsection{Subsystem $H_{A}$}

The subsystem corresponding to $H_{A}$ is

$\dot{\mathbf{X}}=\mathbf{0}, \dot{\mathbf{P}}=-\mathbb{W}^{-1} \frac{\partial H_{A}}{\partial \mathbf{X}}, \dot{\mathbf{S}}=\mathbf{0}$,

$\dot{\mathbf{e}}_{x}=\mathbf{0}, \dot{\mathbf{e}}_{y}=\mathbb{M}_{0}^{-1}\left(\sum_{a=1}^{N_{p}} \omega_{a} \mathbb{N}_{0}\left(x_{a}\right) \mathbf{a}_{y}+\mathbb{C}^{\mathrm{T}} \mathbb{M}_{1} \mathbb{C} \mathbf{a}_{y}\right), \dot{\mathbf{e}}_{z}=\mathbb{M}_{0}^{-1}\left(\sum_{a=1}^{N_{p}} \omega_{a} \mathbb{N}_{0}\left(x_{a}\right) \mathbf{a}_{z}+\mathbb{C}^{\mathrm{T}} \mathbb{M}_{1} \mathbb{C} \mathbf{a}_{z}\right)$,

$\dot{\mathbf{a}}_{y}=\mathbf{0}, \dot{\mathbf{a}}_{z}=\mathbf{0}$.

In this subsystem, $\mathbf{X}, \mathbf{S}, \mathbf{e}_{x}, \mathbf{a}_{y}, \mathbf{a}_{z}$ are unchanged. In the sequel, we will use the identities defined in (3.11) and (3.17)

$\mathbb{N}_{0}\left(x_{a}\right)=\Lambda^{0}\left(x_{a}\right) \Lambda^{0}\left(x_{a}\right)^{\mathrm{T}}, \mathbb{L}:=\frac{\partial}{\partial x_{a}} \mathbb{N}_{0}\left(x_{a}\right)=\frac{\partial}{\partial x_{a}} \Lambda^{0}\left(x_{a}\right) \Lambda^{0}\left(x_{a}\right)^{\mathrm{T}}+\Lambda^{0}\left(x_{a}\right) \frac{\partial}{\partial x_{a}} \Lambda^{0}\left(x_{a}\right)^{\mathrm{T}}$.

Then, for each component of $\mathbf{P}=\left(p_{1}, \ldots, p_{a}, \ldots, p_{N_{p}}\right)$, an explicit Euler integrator becomes exact since $\mathbf{a}_{y}(t)=\mathbf{a}_{y}(0)$ and $\mathbf{a}_{z}(t)=\mathbf{a}_{z}(0)$,

$$
\begin{aligned}
p_{a}(t) & =p_{a}(0)-t \frac{1}{\omega_{a}}\left(\frac{1}{2} \omega_{a} \mathbf{a}_{y}(0)^{\mathrm{T}} \frac{\partial}{\partial x_{a}} \mathbb{N}_{0}\left(x_{a}\right) \mathbf{a}_{y}(0)+\frac{1}{2} \omega_{a} \mathbf{a}_{z}(0)^{\mathrm{T}} \frac{\partial}{\partial x_{a}} \mathbb{N}_{0}\left(x_{a}\right) \mathbf{a}_{z}(0)\right), \\
& =p_{a}(0)-t\left(\frac{1}{2} \mathbf{a}_{y}(0)^{\mathrm{T}} \frac{\partial}{\partial x_{a}} \mathbb{N}_{0}\left(x_{a}\right) \mathbf{a}_{y}(0)+\frac{1}{2} \mathbf{a}_{z}(0)^{\mathrm{T}} \frac{\partial}{\partial x_{a}} \mathbb{N}_{0}\left(x_{a}\right) \mathbf{a}_{z}(0)\right), \\
& =p_{a}(0)-\frac{t}{2} \mathbf{a}_{y}(0)^{\mathrm{T}} \mathbb{L} \mathbf{a}_{y}(0)-\frac{t}{2} \mathbf{a}_{z}(0)^{\mathrm{T}} \mathfrak{L} \mathbf{a}_{z}(0) .
\end{aligned}
$$

For the transverse electric field, we get

$$
\begin{aligned}
& \mathbb{M}_{0} \mathbf{e}_{y}(t)=\mathbb{M}_{0} \mathbf{e}_{y}(0)+t\left(\sum_{a=1}^{N_{p}} \omega_{a} \mathbb{N}_{0}\left(x_{a}\right) \mathbf{a}_{y}(0)+\mathbb{C}^{\mathrm{T}} \mathbb{M}_{1} \mathbb{C} \mathbf{a}_{y}(0)\right), \\
& \mathbb{M}_{0} \mathbf{e}_{z}(t)=\mathbb{M}_{0} \mathbf{e}_{z}(0)+t\left(\sum_{a=1}^{N_{p}} \omega_{a} \mathbb{N}_{0}\left(x_{a}\right) \mathbf{a}_{z}(0)+\mathbb{C}^{\mathrm{T}} \mathbb{M}_{1} \mathbb{C} \mathbf{a}_{z}(0)\right) .
\end{aligned}
$$

REMARK 2. Formula (4.6) turns matrix-vector multiplications into vector-vector multiplications, which will significantly reduce the computational cost.

\subsection{Subsystem $H_{s}$}

The subsystem of ODEs corresponding to $H_{s}=\mathfrak{h} \mathbf{a}_{z}^{\mathrm{T}} \mathbb{C}^{\mathrm{T}} \Lambda_{1}(\mathbf{X})^{\mathrm{T}} \mathfrak{W} \mathbf{S}_{y}-\mathfrak{h} \mathbf{a}_{y}^{\mathrm{T}} \mathbb{C}^{\mathrm{T}} \Lambda_{1}(\mathbf{X})^{\mathrm{T}} \mathfrak{W} \mathbf{S}_{z}$ is

$$
\begin{aligned}
& \dot{\mathbf{X}}=\mathbf{0}, \dot{\mathbf{P}}=-\mathbb{W}^{-1} \frac{\partial H_{s}}{\partial \mathbf{X}}, \dot{\mathbf{S}}=\frac{1}{\mathfrak{h}} \mathbb{S} \frac{\partial H_{s}}{\partial \mathbf{S}} \\
& \dot{\mathbf{e}}_{x}=\mathbf{0}, \dot{\mathbf{e}}_{y}=-\mathfrak{h} \mathbb{M}_{0}^{-1} \mathbb{C}^{\mathrm{T}} \Lambda_{1}(\mathbf{X})^{\mathrm{T}} \mathbb{W} \mathbf{S}_{z}, \dot{\mathbf{e}}_{z}=\mathfrak{h} \mathbb{M}_{0}^{-1} \mathbb{C}^{\mathrm{T}} \Lambda_{1}(\mathbf{X})^{\mathrm{T}} \mathfrak{W} \mathbf{S}_{y}, \\
& \dot{\mathbf{a}}_{y}=\mathbf{0}, \dot{\mathbf{a}}_{z}=\mathbf{0} .
\end{aligned}
$$


For this subsystem, we first solve $\dot{\mathbf{S}}=\frac{1}{\mathfrak{h}} \$ \frac{\partial H_{s}}{\partial \mathbf{S}}$. For the $a$-th particle, we have

$$
\dot{\mathbf{s}}_{a}=\left(\begin{array}{c}
\dot{s}_{a, x} \\
\dot{s}_{a, y} \\
\dot{s}_{a, z}
\end{array}\right)=\left(\begin{array}{ccc}
0 & Y_{a} & Z_{a} \\
-Y_{a} & 0 & 0 \\
-Z_{a} & 0 & 0
\end{array}\right)\left(\begin{array}{c}
s_{a, x} \\
s_{a, y} \\
s_{a, z}
\end{array}\right)=: \hat{r}_{a} \mathbf{s}_{a}
$$

where $Y_{a}=\mathbf{a}_{y}^{\mathrm{T}} \mathbb{C}^{\mathrm{T}} \Lambda^{1}\left(x_{a}\right), Z_{a}=\mathbf{a}_{z}^{\mathrm{T}} \mathbb{C}^{\mathrm{T}} \Lambda^{1}\left(x_{a}\right), \Lambda^{1}\left(x_{a}\right)=\left(\Lambda_{1}^{1}\left(x_{a}\right), \cdots, \Lambda_{N_{1}}^{1}\left(x_{a}\right)\right)^{\mathrm{T}}$. Let us define the vector $\mathbf{r}_{a}=\left(0, Z_{a},-Y_{a}\right) \in \mathbb{R}^{3}$, then the Rodrigues' formula gives the following explicit solution for 4.10

$$
\mathbf{s}_{a}(t)=\exp \left(t \hat{r}_{a}\right) \mathbf{s}_{a}(0)=\left(I+\frac{\sin \left(t\left|\mathbf{r}_{a}\right|\right)}{\left|\mathbf{r}_{a}\right|} \hat{r}_{a}+\frac{1}{2}\left(\frac{\sin \left(\frac{t}{2}\left|\mathbf{r}_{a}\right|\right)}{\frac{\left|\mathbf{r}_{a}\right|}{2}}\right)^{2} \hat{r}_{a}^{2}\right) \mathbf{s}_{a}(0),
$$

where $I$ is the $3 \times 3$ identity matrix.

Next, we integrate in time the equation on the transverse electric field to get (using $\dot{\mathbf{X}}=0)$

$$
\begin{aligned}
& M_{0} \mathbf{e}_{y}(t)=M_{0} \mathbf{e}_{y}(0)-\mathfrak{h} \mathbb{C}^{\mathrm{T}} \Lambda_{1}(\mathbf{X})^{\mathrm{T}} \mathbb{W} \int_{0}^{t} \mathbf{S}_{z}(\tau) \mathrm{d} \tau, \\
& M_{0} \mathbf{e}_{z}(t)=M_{0} \mathbf{e}_{z}(0)+\mathfrak{h} \mathbb{C}^{\mathrm{T}} \Lambda_{1}(\mathbf{X})^{\mathrm{T}} \mathbb{W} \int_{0}^{t} \mathbf{S}_{y}(\tau) \mathrm{d} \tau,
\end{aligned}
$$

where we recall $\mathbf{S}_{i}(\tau)=\left(s_{1, i}, \cdots, s_{a, i}, \cdots, s_{N_{p}, i}\right)^{\mathrm{T}} \in \mathbb{R}^{N_{p}}, i \in\{y, z\}$, see (3.10). We then have to integrate in time the spin variable. This is done using 4.11

$$
\begin{aligned}
\int_{0}^{t} \mathbf{s}_{a}(\tau) \mathrm{d} \tau & =\int_{0}^{t} \exp \left(\tau \hat{r}_{a}\right) \mathbf{s}_{a}(0) \mathrm{d} \tau \\
& =\left(t I-\frac{\cos \left(t\left|\mathbf{r}_{a}\right|\right)}{\left|\mathbf{r}_{a}\right|^{2}} \hat{r}_{a}+\frac{1}{\left|\mathbf{r}_{a}\right|^{2}} \hat{r}_{a}+\frac{2}{\left|\mathbf{r}_{a}\right|^{2}}\left(\frac{t}{2}-\frac{\sin \left(t\left|\mathbf{r}_{a}\right|\right)}{2\left|\mathbf{r}_{a}\right|}\right) \hat{r}_{a}^{2}\right) \mathbf{s}_{a}(0)
\end{aligned}
$$

Now we focus on the impulsion variable $\mathbf{P}$. Using the fact that $\dot{\mathbf{X}}=\mathbf{0}, \dot{\mathbf{a}}_{y}=\dot{\mathbf{a}}_{z}=\mathbf{0}$, we integrate in time each component of $\mathbf{P}$ to get

$$
p_{a}(t)=p_{a}(0)-\mathbf{a}_{z}^{\mathrm{T}}(0) \mathbb{C}^{\mathrm{T}} \frac{\partial \Lambda^{1}\left(x_{a}\right)}{\partial x_{a}} \int_{0}^{t} s_{a, y}(\tau) \mathrm{d} \tau+\mathbf{a}_{y}^{\mathrm{T}}(0) \mathbb{C}^{\mathrm{T}} \frac{\partial \Lambda^{1}\left(x_{a}\right)}{\partial x_{a}} \int_{0}^{t} s_{a, z}(\tau) \mathrm{d} \tau .
$$

\subsection{Subsystem $H_{E}$}

The subsystem corresponding to $H_{E}=\frac{1}{2} \mathbf{e}_{x}^{\mathrm{T}} \mathbb{M}_{1} \mathbf{e}_{x}+\frac{1}{2} \mathbf{e}_{y}^{\mathrm{T}} \mathbf{M}_{0} \mathbf{e}_{y}+\frac{1}{2} \mathbf{e}_{z}^{\mathrm{T}} \mathbb{M}_{0} \mathbf{e}_{z}$ is

$$
\begin{aligned}
& \dot{\mathbf{X}}=\mathbf{0}, \dot{\mathbf{P}}=\Lambda_{1}(\mathbf{X}) \mathbf{e}_{x}, \dot{\mathbf{S}}=\mathbf{0}, \\
& \dot{\mathbf{e}}_{x}=\mathbf{0}, \dot{\mathbf{e}}_{y}=\mathbf{0}, \dot{\mathbf{e}}_{z}=\mathbf{0}, \\
& \dot{\mathbf{a}}_{y}=-\mathbf{e}_{y}, \dot{\mathbf{a}}_{z}=-\mathbf{e}_{z} .
\end{aligned}
$$

Since $\dot{\mathbf{X}}=\mathbf{0}, \dot{\mathbf{e}}_{x}=\mathbf{0}$, the equation on $\mathbf{P}$ can be solved easily

$$
\mathbf{P}(t)=\mathbf{P}(0)+t \Lambda_{1}(\mathbf{X}) \mathbf{e}_{x}(0) .
$$

Similarly, the transverse vector potential can be computed exactly in time:

$$
\mathbf{a}_{y}(t)=\mathbf{a}_{y}(0)-t \mathbf{e}_{y}(0), \quad \mathbf{a}_{z}(t)=\mathbf{a}_{z}(0)-t \mathbf{e}_{z}(0) .
$$




\section{Numerical simulations of stimulated Raman scattering with polarized electrons}

This part is devoted to numerical simulations of a laser-plasma model using the geometric PIC method detailed in the preceding sections. Laser-plasma interactions play a decisive role in many areas of plasma physics, most notably inertial fusion and plasma accelerators. Recently, the interaction of an intense laser pulse with a polarized electron beam was studied numerically using PIC methods (Wen et al. 2017, 2019). In these works, different models were developed based either on classical considerations or on the semiclassical limit of the Dirac Hamiltonian in the Foldy-Wouthuysen representation. The electrons' emission of radiation was taken into account via the Landau-Lifshitz force.

One of the most topical problems in laser-plasma interactions is the efficient acceleration of charged particles (electrons) by large-amplitude longitudinal plasma waves. An efficient way to create such large-amplitude plasma waves involves the stimulated Raman scattering (SRS) mechanism (Forslund et al. 1975), which can be viewed as a parametric instability. During SRS, an incident electromagnetic wave decays into a scattered electromagnetic wave and a Langmuir (plasma) wave, which is responsible for the electron acceleration (see figure 1). Our purpose is to investigate the effect of the electron spin on the SRS instability.

As the full system is rather complex, we will proceed step by step. First, we consider the SRS problem without spin, which was studied in several past works using a gridbased (Eulerian) Vlasov code (Huot et al. 2003, Ghizzo et al. 1990, Li et al. $2019 b$ ). As Eulerian codes are particularly stable and accurate over the entire phase space, we will use them as a benchmark for our PIC simulations. The benchmark will be carried out in the spin-less case, for which an Eulerian code is available. Second, we consider the spin Vlasov-Maxwell model studied in Sec. 2.2 but remove the effect of the plasma on the propagation of the electromagnetic wave. This amounts to assuming that the wave propagates freely (as in vacuum) and interacts with the plasma, but the plasma does not impact the propagation of the wave. In contrast, the longitudinal nonlinearity due to Poisson's equation is maintained. In this case, an approximate solution of the spin dynamics can be obtained analytically, which enables us to validate the numerical simulations. Finally, we simulate the complete spin-dependent model (2.7) and study the influence of the various physical parameters: amplitude of the electromagnetic wave, temperature, initial electron polarization, and scaled Planck constant.

\subsection{SRS without spin}

We consider the model put forward in Huot et al. (2003); Ghizzo et al. $(1990)$; Li et al. (2019b), which corresponds to our equations (2.7) where the spin dependence has been removed. The distribution function $f(t, x, p)$ and the electromagnetic fields 


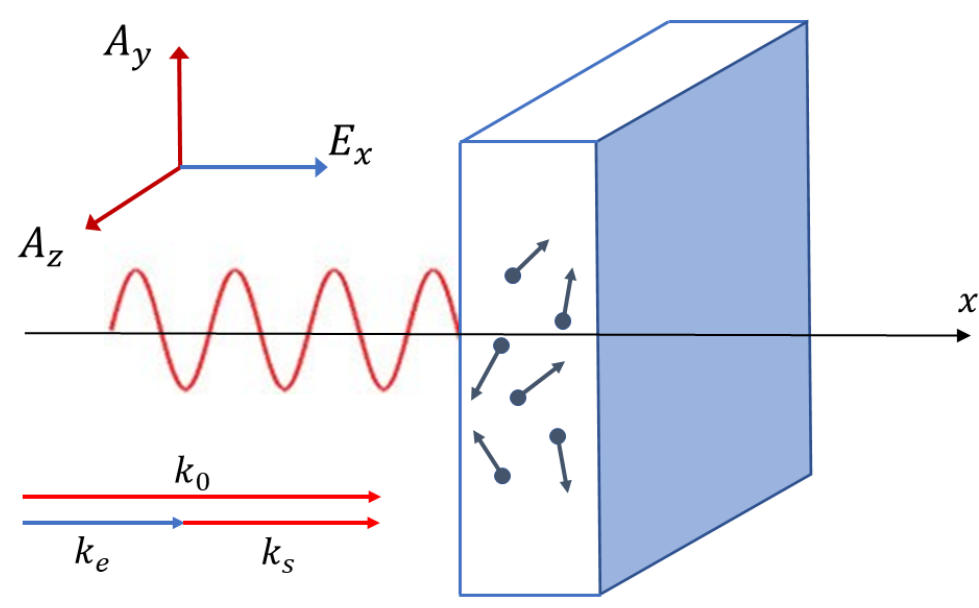

FigURE 1. Schematic view of the geometry of the laser-plasma interaction during stimulated Raman scattering.

$\left(E_{x}, \mathbf{E}_{\perp}, \mathbf{A}_{\perp}\right)(t, x)$ obey the equations

$$
\begin{aligned}
& \frac{\partial f}{\partial t}+p \frac{\partial f}{\partial x}+\left[E_{x}-\mathbf{A}_{\perp} \cdot \frac{\partial \mathbf{A}_{\perp}}{\partial x}\right] \frac{\partial f}{\partial p}=0, \\
& \frac{\partial E_{x}}{\partial t}=-\int_{\mathbb{R}} p f \mathrm{~d} p \\
& \frac{\partial E_{y}}{\partial t}=-\frac{\partial^{2} A_{y}}{\partial x^{2}}+A_{y} \int_{\mathbb{R}} f \mathrm{~d} p, \\
& \frac{\partial E_{z}}{\partial t}=-\frac{\partial^{2} A_{z}}{\partial x^{2}}+A_{z} \int_{\mathbb{R}} f \mathrm{~d} p \\
& \frac{\partial A_{y}}{\partial t}=-E_{y} \\
& \frac{\partial A_{z}}{\partial t}=-E_{z} .
\end{aligned}
$$

As initial condition for $f$, we use a perturbed Maxwellian

$$
f(t=0, x, p)=\left(1+\alpha \cos \left(k_{e} x\right)\right) \frac{1}{\sqrt{2 \pi} v_{t h}} e^{-\frac{p^{2}}{2 v_{t h}^{2}}}, \quad x \in[0, L], p \in \mathbb{R},
$$

so that the initial longitudinal electric field is: $E_{x}(t=0, x)=\frac{\alpha}{k_{e}} \sin \left(k_{e} x\right)$. Here, $\alpha$ and $k_{e}$ are the amplitude and the wave number of the perturbation, respectively, and $v_{t h}$ is the electron thermal speed. For the transverse fields, we consider an electromagnetic wave with circular polarization:

$$
\begin{aligned}
& E_{y}(t=0, x)=E_{0} \cos \left(k_{0} x\right), \quad E_{z}(t=0, x)=E_{0} \sin \left(k_{0} x\right), \\
& A_{y}(t=0, x)=-\frac{E_{0}}{\omega_{0}} \sin \left(k_{0} x\right), A_{z}(t=0, x)=\frac{E_{0}}{\omega_{0}} \cos \left(k_{0} x\right),
\end{aligned}
$$


where $k_{0}$ is the wave number and $E_{0}$ is the amplitude of the transverse electric field. We use periodic boundary conditions with spatial period $L=4 \pi / k_{e}$. The circular polarization is chosen because it is likely to have maximum impact on the electron spin, which will be considered in the next two subsections.

In the SRS instability, the incident electromagnetic wave $\left(\omega_{0}, k_{0}\right)$ drives two waves inside the plasma: a scattered electromagnetic wave $\left(\omega_{s}, k_{s}\right)$ and an electron plasma wave $\left(\omega_{e}, k_{e}\right)$, where $\omega$ and $k$ denote respectively the frequency and wave number of each wave. These waves are matched according to the conditions (conservation of energy and momentum):

$$
\omega_{0}=\omega_{s}+\omega_{e}, \quad \text { and } \quad k_{0}=k_{s}+k_{e},
$$

with $\omega_{0, s}^{2}=1+k_{0, s}^{2}$ and $\omega_{e}^{2}=1+3 v_{t h}^{2} k_{e}^{2}$.

A schematic view of this configuration is shown in figure 1 .

Following Ghizzo et al. (1990), we take the following values for the physical parameters of the plasma and the wave (recall that frequencies are normalized to $\omega_{p}$, velocities to $c$, wave numbers to $\omega_{p} / c$, and electric fields to $\left.\omega_{p} m c / e\right)$ :

$$
\alpha=0.02, \quad k_{e}=1.22, \quad k_{0}=2 k_{e}, \quad v_{t h}=0.17 .
$$

Using the matching conditions (5.4), this yields:

$$
k_{s}=k_{e}, \quad \omega_{0}=2.63, \quad \omega_{s}=1.562, \quad \omega_{e}=1.061 .
$$

As a reference value, we take for the amplitude of the incident wave $E_{\text {ref }}=0.325$. The actual values used in the simulations will be in the range $0.25 E_{\text {ref }} \leqslant E_{0} \leqslant 2 E_{\text {ref }}$ In all cases, the quiver velocity $v_{o s c}=E_{0} / \omega_{0}$ is smaller than unity, which ensures that the present non-relativistic approximation is valid.

In order to check the accuracy of our PIC code in the spin-less regime, we also developed a grid-based Eulerian code (see ( $\mathrm{Li}$ et al. 2019b) for more details). For the PIC code, the numerical parameters are as follows: $N_{x}=512, N_{\text {part }}=5 \times 10^{5}, \Delta t=0.01$, whereas for the grid code we take: $N_{x}=128, N_{v}=128, \Delta t=0.01$. Both codes preserve the total energy with a relative error less than $0.05 \%$.

In figure 2, we plot the time evolutions of the longitudinal electric field norm

$$
\left\|E_{x}(t)\right\|=\left(\frac{1}{2} \int_{0}^{L} E_{x}^{2} \mathrm{~d} x\right)^{1 / 2}
$$

(in semi- $\log _{e}$ scale) for two values of the incident wave amplitude $\left(E_{0}=0.325\right.$ and $\left.E_{0}=0.65\right)$. The initial conditions are the same for both codes, but the PIC code displays a higher level of initial fluctuations (noise) that is inherent to the numerical method. This is also why we had to take a somewhat high initial perturbation $(\alpha=0.02)$, so that it is significantly larger than the noise level. We can observe a relatively good agreement between these two numerical methods in the linear phase. The observed growth rate $\left(\gamma \approx 0.03\right.$ for $E_{0}=0.325$ and $\gamma \approx 0.06$ for $\left.E_{0}=0.65\right)$ is proportional to the amplitude $E_{0}$ and close to the value expected from the linear theory $(\gamma \approx 0.04$, see (Ghizzo et al. 1990).

\subsection{Spin-dependent model without wave self-consistency}

In this part, we study the propagation of a circularly polarized wave into a plasma which does not retroact on the wave. Hence, the electromagnetic wave propagates as if it was in vacuum. In this case, there is no SRS instability. 


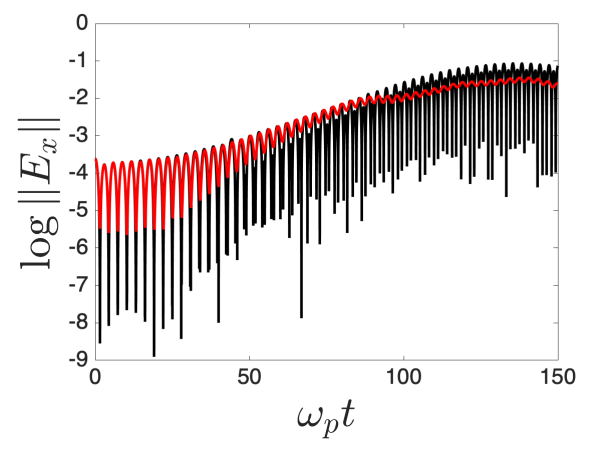

(a)

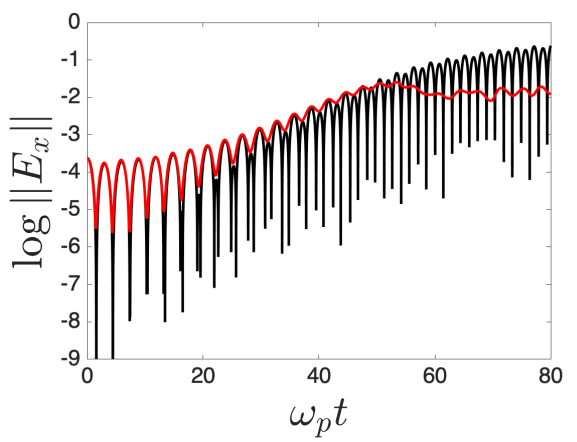

(b)

FiguRE 2. SRS simulations without spin. Time evolution of the amplitude of the longitudinal electric field norm $\left\|E_{x}(t)\right\|$ on a semi- $\log _{e}$ scale. We compare the results of the Eulerian grid code (black curves) and the PIC code (red curves), for two values of the initial amplitude of the electromagnetic wave. Left frame: $E_{0}=E_{\text {ref }}=0.325$ (observed slope in the linear regime: $\gamma \approx 0.03)$. Right frame: $E_{0}=2 E_{\mathrm{ref}}=0.65(\gamma \approx 0.06)$.

With the notations of Sec. 2.2, the model we consider here reads

$$
\dot{\mathcal{Z}}=\{\mathcal{Z}, \widetilde{\mathcal{H}}\}
$$

where $\mathcal{Z}=\left(f, E_{x}, E_{y}, E_{z}, A_{y}, A_{z}\right),\{\cdot, \cdot\}$ is the bracket defined in 2.8 and $\tilde{\mathcal{H}}$ is the Hamiltonian defined as

$$
\begin{aligned}
\widetilde{\mathcal{H}}\left(f, \mathbf{E}, \mathbf{A}_{\perp}\right)= & \frac{1}{2} \int_{\mathbb{R}^{5}} p^{2} f \mathrm{~d} x \mathrm{~d} p \mathrm{~d} \mathbf{s}+\frac{1}{2} \int_{\mathbb{R}}\left(|\mathbf{E}|^{2}+\left|\frac{\partial \mathbf{A}_{\perp}}{\partial x}\right|^{2}\right) \mathrm{d} x \\
& +\mathfrak{h} \int_{\mathbb{R}^{5}}\left(s_{y} \frac{\partial A_{z}}{\partial x}-s_{z} \frac{\partial A_{y}}{\partial x}\right) f \mathrm{~d} x \mathrm{~d} p \mathrm{~d} \mathbf{s},
\end{aligned}
$$

which means that the term $\mathbf{A}_{\perp} \cdot \frac{\partial \mathbf{A}_{\perp}}{\partial x} \frac{\partial f}{\partial p}$ disappears from the Vlasov equation in (2.7), and the terms $A_{y} \int_{\mathbb{R}^{4}} f \mathrm{~d} p \mathrm{~d} \mathbf{s}, A_{z} \int_{\mathbb{R}^{4}} f \mathrm{~d} p \mathrm{~d} \mathbf{s}$ disappear from the Maxwell equations in (2.7). In this case, the electromagnetic wave is not coupled to the plasma and can be determined exactly by solving the corresponding Maxwell equations for $E_{y}$ and $E_{z}$. In contrast, the longitudinal nonlinearity is kept in the model, hence $E_{x}$ is a solution of Poisson's equation. The resulting reduced system preserves the geometric structure of the full system.

The initial condition on the distribution function is as follows (see Appendix D):

$$
f(t=0, x, p, \mathbf{s})=\frac{1}{4 \pi}\left(1+\eta s_{z}\right)\left(1+\alpha \cos \left(k_{e} x\right)\right) \frac{e^{-\frac{p^{2}}{2 v_{t h}^{2}}}}{\sqrt{2 \pi} v_{t h}}, x \in\left[0, \frac{4 \pi}{k_{e}}\right], p \in \mathbb{R}, \mathbf{s} \in \mathbb{S}^{2},
$$

ie, it is the product of the initial spin-less distribution function 5.2 times a spindependent part. Hence, the spin variables are initially uncorrelated with the positions and velocities of the particles. The spin-dependent part should be fully quantum mechanical, and is therefore obtained from the $2 \times 2$ density matrix for spin- $1 / 2$ fermions, with the additional assumption that the spin must be directed either parallel or antiparallel to the $z$ axis (Manfredi et al. 2019) (collinear approximation). More details are given in the Appendix $\mathrm{D}$. The variable $\eta$ represents the degree of polarization of the electron gas: $\eta=0$ for an unpolarized gas and $\eta=1$ for a fully polarized one. Here, we use $\eta=0.5$ and $\mathfrak{h}=0.00022$. This value of the scaled Planck constant corresponds to a dense electron 
gas of density $n_{0}=10^{31} \mathrm{~m}^{-3}$ and temperature $k_{B} T=100 \mathrm{eV}$. All other parameters take the same values as in the spin-less case treated in Sec. 5.1.

Neglecting the spatial dependence of the magnetic fields, an approximate closed equation for the dynamics of the macroscopic spin $\mathbf{S}(t)=\int \mathbf{s} f \mathrm{~d} x \mathrm{~d} p \mathrm{~d} \mathbf{s} \in \mathbb{R}^{3}$ can be obtained by integrating the Vlasov equation (2.7) in $(x, p, \mathbf{s})$. We get the following ODE system

$$
\dot{\mathbf{S}}=\mathbf{S} \times \mathbf{B},
$$

with $\mathbf{B}=\left(0,-\partial_{x} A_{z}, \partial_{x} A_{y}\right)^{\mathrm{T}}$. Considering a circularly polarized electromagnetic wave, the magnetic field is (still neglecting spatial effects) $\mathbf{B}(t)=\left(0, B_{0} \sin \left(\omega_{0} t\right),-B_{0} \sin \left(\omega_{0} t\right)\right.$, with $B_{0}=E_{0} k_{0} / \omega_{0}$. In the regime $B_{0} / \omega_{0} \ll 1$ (ie, when the Larmor precession frequency $e B_{0} / m$ is much smaller than the laser frequency), it can be shown that the spin $S_{z}(t)$ oscillates with a frequency $\omega_{\text {spin }}=B_{0}^{2} /\left(2 \omega_{0}\right)$ (see Appendix C for details).

Here, this solution will be compared to the evolution of $\mathbf{S}(t)$ obtained from the numerical solution of the spin Vlasov-Maxwell equations. As the retroaction terms were removed from the Vlasov equation, there is no SRS instability. Then, the magnetic field of the incident wave remains approximately constant in amplitude, thus justifying the use of (5.7) as a valid approximation.

We use the same physical parameters as in Sec. 5.1 and define the reference magnetic field of the incident wave as

$$
B_{\mathrm{ref}}=\frac{E_{\mathrm{ref}} k_{0}}{\omega_{0}}=0.123 k_{0}, \quad \text { so that } \quad \frac{B_{\mathrm{ref}}^{2}}{2 \omega_{0}}=0.017 .
$$

We have that $B_{\text {ref }} / \omega_{0}=0.123 k_{0} / \omega_{0} \approx 0.11$. We will use values up to $B_{0}=2 B_{\text {ref }}$, so that the condition $B_{0} / \omega_{0} \ll 1$ is always satisfied, albeit marginally.

In figure 3 , we plot the time evolution of the $z$ component of the macroscopic spin vector $S_{z}(t)=\int s_{z} f \mathrm{~d} x \mathrm{~d} p \mathrm{~d}$ s and its frequency spectrum (the frequencies are expressed in units of $2 \pi / T$, where $T$ is the final simulation time), for three different value of $E_{0}$ (and hence $\left.B_{0}\right): E_{0}=0.5 E_{\text {ref }}, E_{0}=E_{\text {ref }}$, and $E_{0}=2 E_{\text {ref. }} S_{z}$ displays a damped oscillatory behaviour, with a spectrum that is well-localized around a single frequency. This dominant frequency is close to the theoretical value $\omega_{\text {spin }}=B_{0}^{2} /\left(2 \omega_{0}\right)$, which yields $\omega_{\text {spin }}=0.0043,0.018$, and 0.068 for the three cases considered here. In particular, the quadratic scaling between $\omega_{\text {spin }}$ and $B_{0}$ is well respected.

The observed damping is likely to be due to phase mixing in the phase space and its rate also increases rapidly with $E_{0}$. The effect of the temperature on the damping will be studied in more details in the next subsection.

\subsection{Full spin-dependent model}

In this section, we consider the full spin Vlasov-Maxwell model 2.7 and study the influence of several physical parameters on the spin dynamics. In particular, we will analyze the effect of the incident wave amplitude, the electronic temperature, the initial spin polarization, and the scaled Planck constant. The remaining physical parameters are those of Sec. 5.1

We will consider two types of initial conditions for the distribution function. The first (labeled 'Wigner') is the one already used in the preceding subsection, see (5.6), and corresponds to a quantum density matrix for an ensemble of spins directed parallel to the $z$ axis. The second initial condition (labeled 'Dirac') corresponds to an ensemble of classical spins all having directions along the $z$ axis; it does not correspond to any quantum state and is used here only to illustrate more clearly the loss of spin polarization in the electron gas. 


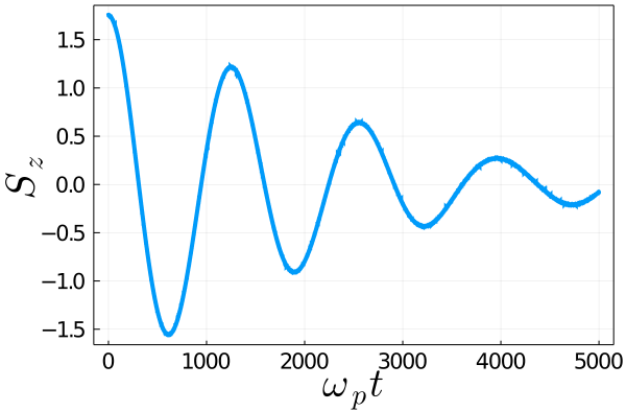

(a)

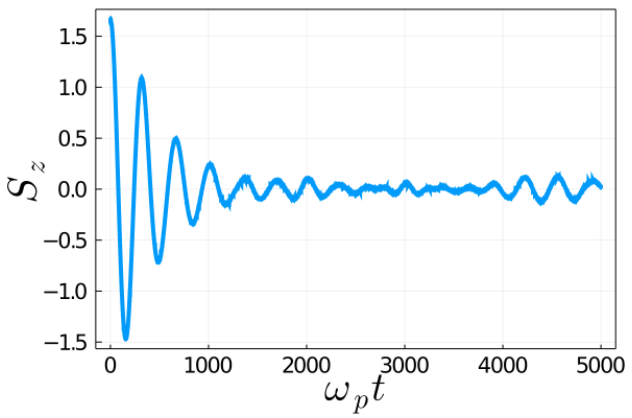

(c)

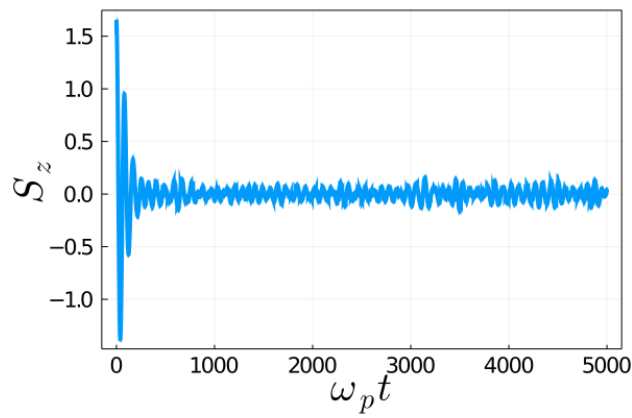

(e)

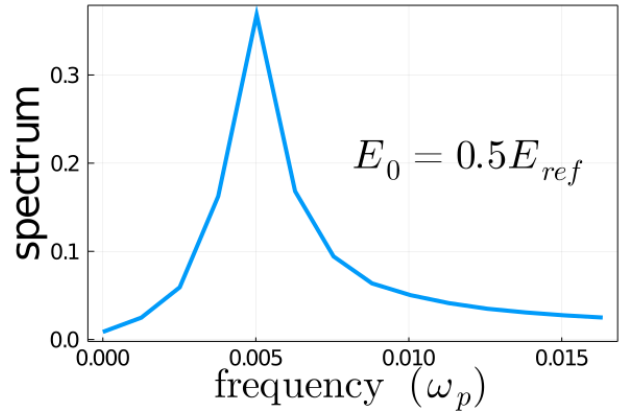

(b)

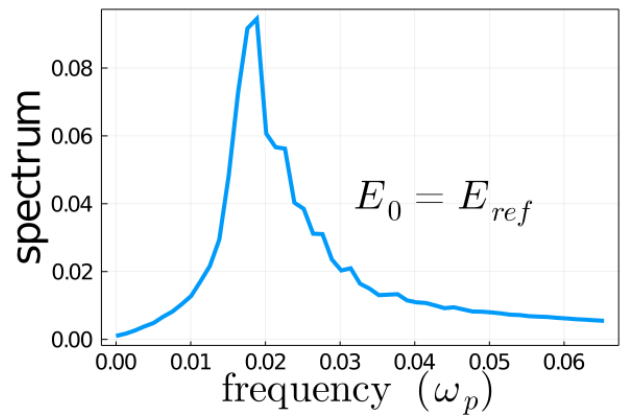

(d)

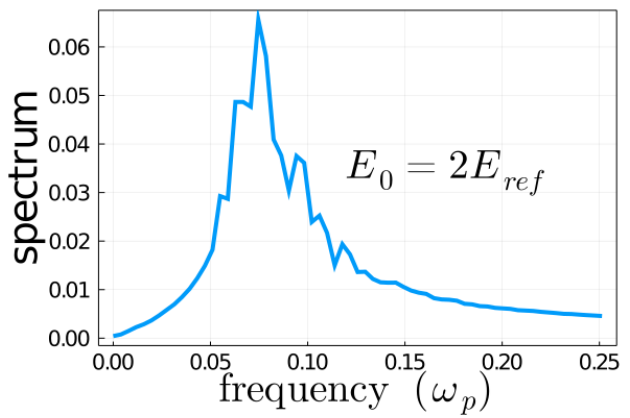

(f)

FiguRE 3. Spin-dependent model without wave self-consistency, for three values of the incident wave amplitude: $E_{0}=0.5 E_{\text {ref }}$ (top), $E_{0}=E_{\text {ref }}$ (middle), and $E_{0}=2 E_{\text {ref }}$ (bottom). Left panels: time history of $S_{z}(t)$ (time is in units of $\omega_{p}^{-1}$ ). Right panels: corresponding frequency spectrum, in units of $\omega_{p}$. The expected values of the spin precession frequencies are (from top to bottom): $\omega_{\text {spin }}=0.0043,0.018$, and 0.068 .

\subsubsection{Wigner initial condition}

The initial condition is the Maxwell-Boltzmann distribution of equation (5.6), whose derivation is detailed in the Appendix D. The physical parameters are the same as in our reference case described in Sec. 5.1. The electromagnetic fields are initialized as in equation (5.3). The numerical parameters are as follows: $\Delta t=0.04, N_{p}=2 \times 10^{4}, N_{x}=$ 128.

Laser field amplitude. In figure 4, we study the influence of the incident wave amplitude $E_{0}$, using $\mathfrak{h}=0.00022$. The two columns in the figure refer to two values of $E_{0}: E_{\text {ref }}$ 
on the left and $2 E_{\text {ref }}$ on the right. Conservation of the total energy is good for PIC code standards, with an error on the relative energy $\left|\mathcal{E}_{\text {tot }}(t)-\mathcal{E}_{\text {tot }}(0)\right| / \mathcal{E}_{\text {tot }}(0)$ of about $3 \times 10^{-4}$. This good conservation property is due to the fact that our algorithm is a fully discrete structure-preserving method.

The instability rate, measured from the growth of the longitudinal electric field amplitude $\left\|E_{x}(t)\right\|$, is very similar to the one observed in the spin-less simulations, namely $\gamma=0.03\left(E_{0}=E_{\text {ref }}\right)$ and $\gamma=0.06\left(E_{0}=2 E_{\text {ref }}\right)$.

The instability has a strong impact on the spin dynamics. In particular, the $z$ component of the macroscopic spin $S_{z}(t)=\int s_{z} f \mathrm{~d} x \mathrm{~d} p \mathrm{~d}$ s is damped much more efficiently in the large-amplitude case (right column), for which the instability develops faster and saturates at a higher level. The frequency spectrum of $S_{z}(t)$ displays in both cases a well-defined peak. There is a factor of about 5.5 between the spin precession frequencies observed for $E_{0}=E_{\text {ref }}$ and $E_{0}=2 E_{\text {ref }}$. This is slightly larger that the quadratic scaling with $E_{0}$ predicted by our simple model $\omega_{\text {spin }}=B_{0}^{2} /\left(2 \omega_{0}\right)$ (see Sec. 5.2 and Appendix $\mathrm{C}$ for further details). However, one must recall that this expression was derived under the assumption of an incident wave of constant amplitude $E_{0}$. Here, in contrast, the amplitude is modulated in time, which then affects the quadratic scaling.

Thermal effects. Next, we study the influence of the temperature, by considering two values of the thermal speed: $v_{t h}=0.17$ (as in the previous results of figure 3) and $v_{t h}=0.51$, while the field amplitude is fixed to $E_{0}=E_{\text {ref }}$ and $\mathfrak{h}=0.00022$. The wave number $k_{e}$ also changes, as it is dependent on the temperature $\left(k_{e}=1.22\right.$ and $k_{e}=1.46$, for the low-temperature and high-temperature cases, respectively), but the matching conditions (5.4) are still satisfied with $k_{0}=2 k_{e}$ and $\omega_{0}^{2}=1+k_{0}^{2}$.

The simulation results are shown in figure 5. In the high-temperature case, the instability is clearly suppressed, but nevertheless the spin is damped much more quickly than in the 'cold' case. On the other hand, we checked that when no laser pulse is present at all (ie, $E_{0}=0$, not shown here) no loss of the polarization is observed.

This is an intriguing result. First, it reveals that the laser pulse is mandatory to induce some loss of polarization. When the laser wave amplitude $E_{0}$ is sufficiently high and the plasma sufficiently cold, the SRS instability is triggered, and this induces a loss of spin polarization in the electron gas. This loss of polarization is faster for larger wave amplitudes (figure 4). For warmer plasmas, the SRS instability is suppressed, but depolarization still occurs due to temperature effects (figure 5). Nevertheless, the presence of the electromagnetic wave is still necessary to observe this 'thermal' loss of polarization.

Such depolarization mechanisms are akin to the ultrafast demagnetization observed in metallic nanostructures irradiated with femtosecond laser pulses (Beaurepaire et al. 1996: Bigot et al. 2009; Bigot \& Vomir 2013).

Scaled Planck constant and initial polarization. In figure 6, the time history of $S_{z}(t)$ is displayed for $\eta=0.5$ and different values of the scaled Planck constant: $\mathfrak{h}=0.00022,0.05,0.1$, and 0.2 . The influence of $\mathfrak{h}$ on the spin dynamics is rather modest, and becomes appreciable only for values that are approaching unity. The main observable effect is an increase of the oscillation period with the scaled Planck constant.

Figure 7 shows the influence of the initial electron polarization $(\eta=0.2,0.5$, and 1$)$ for two different values of the scaled Planck's constant, $\mathfrak{h}=0.00022$ and $\mathfrak{h}=0.2$. The initial value of the macroscopic spin $S_{z}$ is given by $S_{z}(t=0)=L\left\langle s_{z}\right\rangle=L \eta / 3 \approx 3.42 \eta$, see also the Appendix $\mathrm{D}$. Hence, in order to compare results with different values of $\eta$, we plot the quantity $S_{z}(t) / \eta$. At low $\mathfrak{h}$, the effect of $\eta$ is weak. In contrast, for large $\mathfrak{h}$, a larger initial polarization is associated with a stronger damping of the macroscopic spin.

Spin dynamics. In order to investigate the microscopic spin dynamics in some more details, we have divided the interval of values taken by the spin component $s_{z} \in[-1,+1]$ 


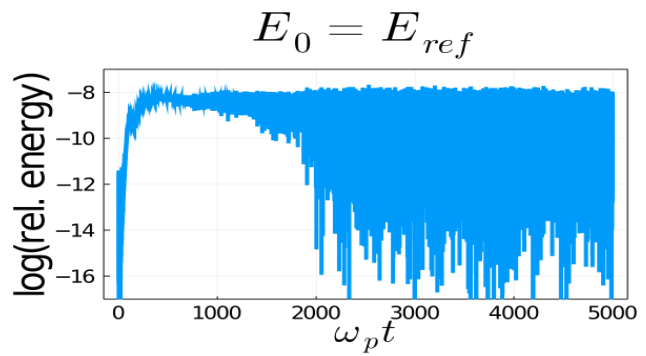

(a)

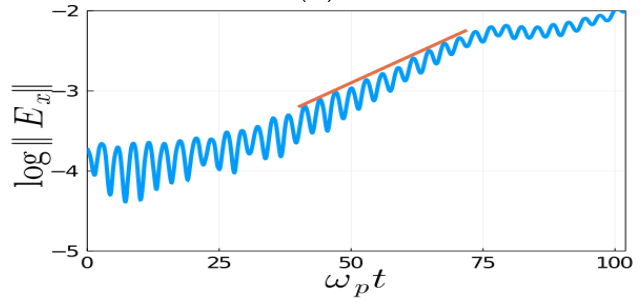

(c)

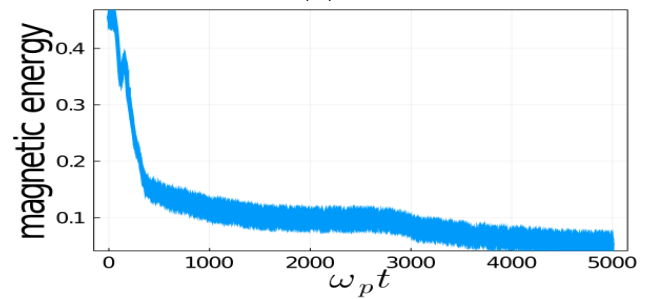

(e)

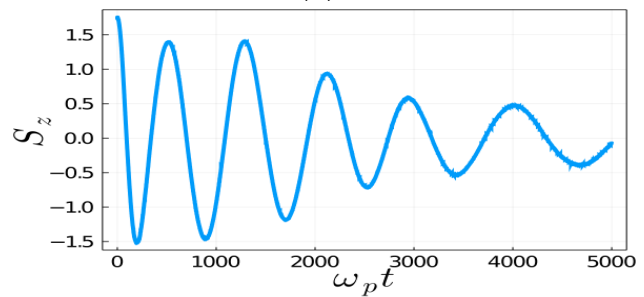

(g)

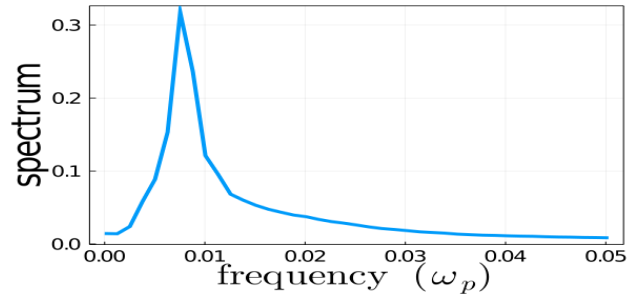

(i)

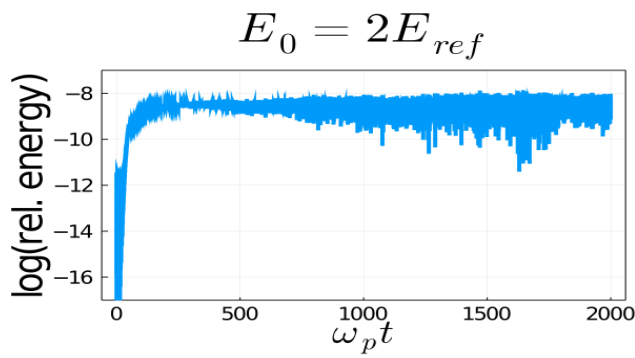

(b)

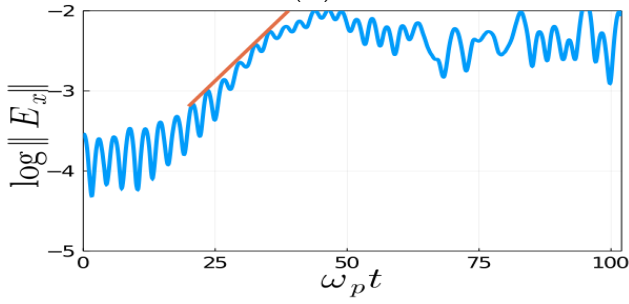

(d)

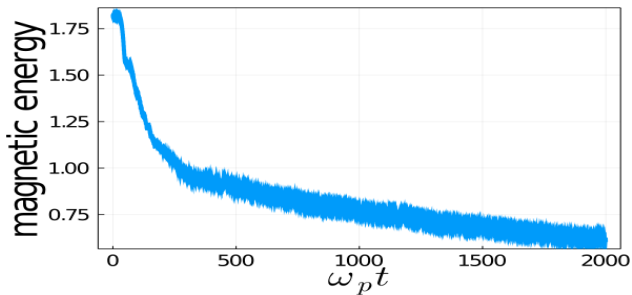

(f)

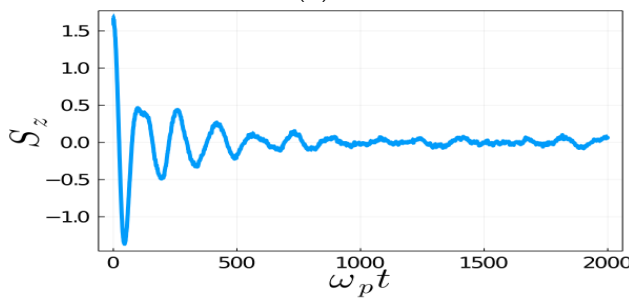

(h)

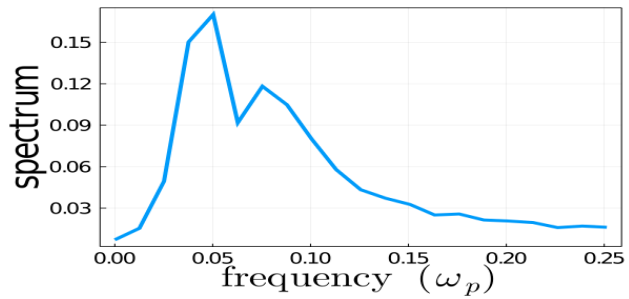

(j)

Figure 4. Full spin-dependent model. We illustrate the influence of the amplitude $E_{0}$ of the incident wave. Left column $E_{0}=E_{\text {ref }}$; right column $E_{0}=2 E_{\text {ref }}$. First line from top: time history of the the relative total energy $\left|\mathcal{E}_{\text {tot }}(t)-\mathcal{E}_{\text {tot }}(0)\right| / \mathcal{E}_{\text {tot }}(0)$ (semi- $\log _{e}$ scale). Second line: time history of the longitudinal electric field norm $\left\|E_{x}\right\|$ (semi- $\log _{e}$ scale); the red straight lines have slopes 0.03 (left) and 0.06 (right). Third line: magnetic energy $\frac{1}{2} \int|B|^{2} d x$. Fourth line: time history of the spin component $S_{z}(t)$. Bottom line: frequency spectrum of $S_{z}$. 

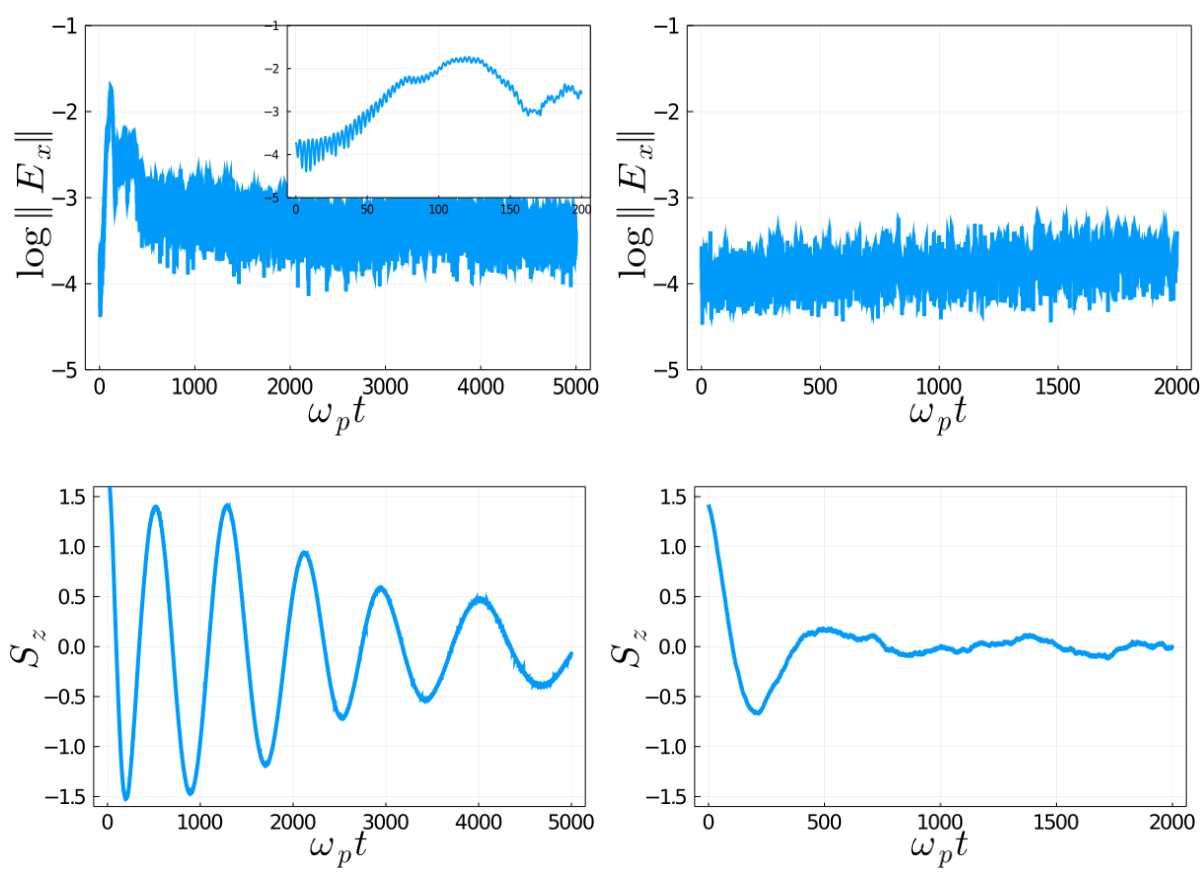

FiguRE 5. Full spin-dependent model. Influence of the electron temperature using two values of the thermal speed: $v_{t h}=0.17$ (left column) and $v_{t h}=0.51$ (right column). For both cases, the field amplitude is $E_{0}=E_{\text {ref }}$. The top panels show the time history of the longitudinal electric field norm (semi- $\log _{e}$ scale). The bottom panels show the time history of the spin component $S_{z}(t)$. The inset in the top left panel displays a zoom of the longitudinal electric field evolution in the time range $\omega_{p} t \in[0,200]$, showing the development of the linear instability.

into 200 bins of size 0.01 each. We call $N\left(s_{z}\right)$ the number of particles having the spin component $s_{z}$ in an interval around $s_{z}$, and $N_{p}$ the total number of particles. In figure $8(\mathrm{a})$-(k), we show the histograms of $N\left(s_{z}\right) / N_{p}$ as a function of $s_{z}$ at different times. The electron distribution at $t=0$ is linear in $s_{z}$, as shown in equation (5.6) and in the Appendix D. In this case, the electron gas is initially fully polarized along the positive $z$ direction, so that the distribution goes from $N\left(s_{z}\right) / N_{p}=0$ for $s_{z}=-1$ to $N\left(s_{z}\right) / N_{p}=1$ for $s_{z}=1$.

For instance, one can see that at $\omega_{p} t=200$ [figure $8(\mathrm{c})$ ] the spin direction has completely reversed, indicating that the global spin now points along the negative $z$ direction. In contrast, at $\omega_{p} t=1500$ [figure 8(i)] the distribution is flat, indicating that the global spin is close to zero. The slope of the spin distribution decreases progressively with time, thus revealing some amount of damping. This behaviour is confirmed by the time history of the global spin component $S_{z}(t)$, shown in figure $8(1)$.

\subsubsection{Dirac initial condition}

We consider the following initial condition for the distribution function

$f_{0}(x, p, \mathbf{s})=\left(1+\alpha \cos \left(k_{e} x\right)\right) \frac{1}{\sqrt{2 \pi} v_{t h}} e^{-\frac{p^{2}}{2 v_{t h}^{2}}} \delta\left(\mathbf{s}-(0,0,1)^{\mathrm{T}}\right), \quad x \in\left[0, \frac{4 \pi}{k_{e}}\right], p \in \mathbb{R}, \mathbf{s} \in \mathbb{S}^{2}$,

which corresponds to an ensemble of classical spins which are all directed along the positive $z$ axis. This distribution does not have a clear quantum-mechanical meaning, as it does not correspond to any actual Wigner function or density matrix. However, it is 


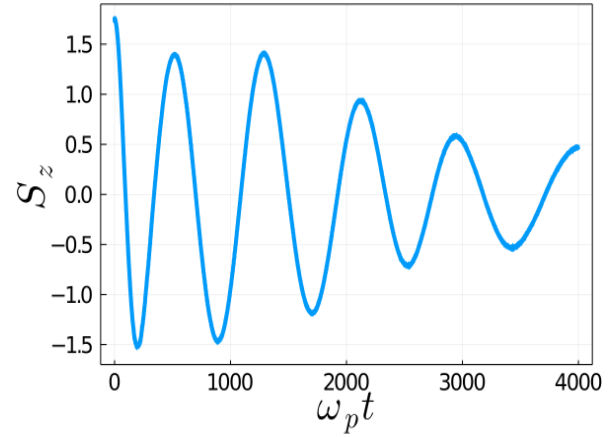

(a)

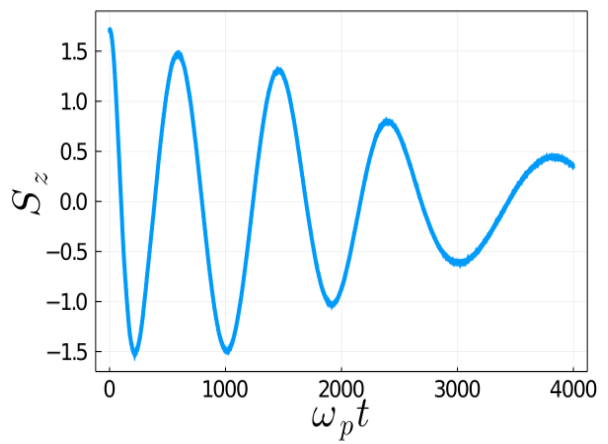

(c)

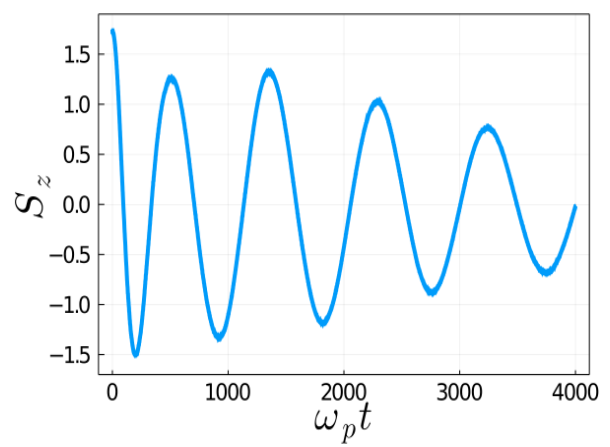

(b)

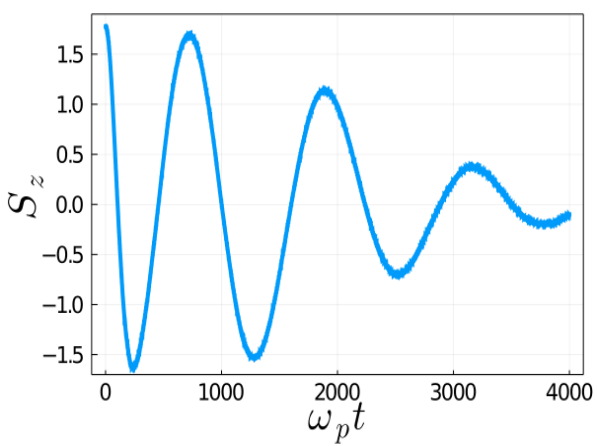

(d)

FiguRE 6. Full spin-dependent model. Influence of $\mathfrak{h}$ on $S_{z}(t)$ : (a) $\mathfrak{h}=0.00022 ;$ (b) $\mathfrak{h}=0.05$; (c) $\mathfrak{h}=0.1 ;$ (d) $\mathfrak{h}=0.2$. For all cases, the initial spin polarization is $\eta=0.5$.

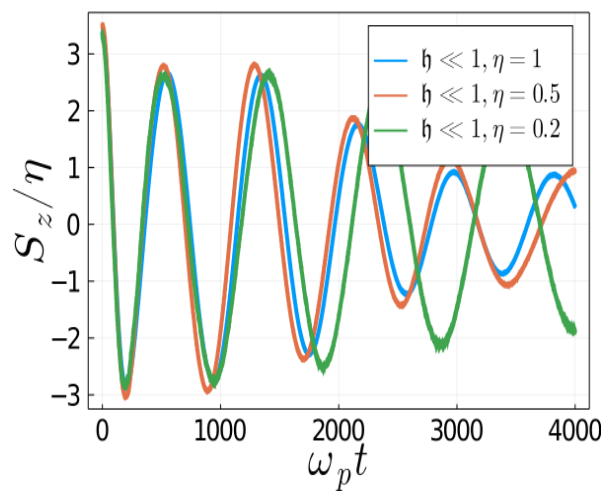

(a)

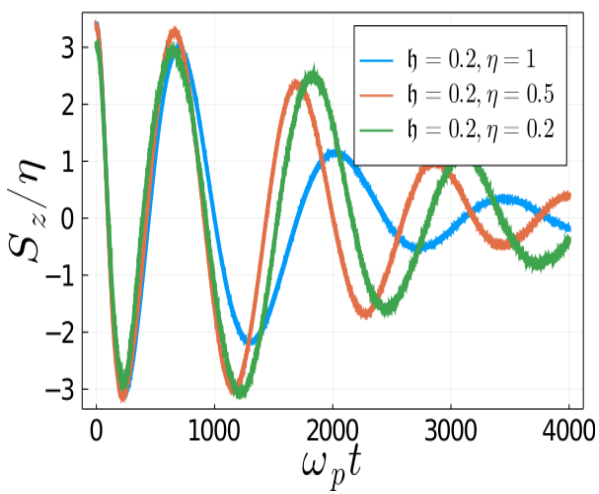

(b)

Figure 7. Full spin dependent model. Influence of the initial polarization $\eta$ for two values of the scaled Planck constant $\mathfrak{h}$. We plot the ratio $S_{z}(t) / \eta$, which is initially independent of $\eta$. (a) $\mathfrak{h}=0.00022, \eta=0.2,0.5$, and $1 ;(b) \mathfrak{h}=0.2, \eta=0.2,0.5$, and 1 .

useful to perform some numerical simulations with this initial condition, as it enables us to better highlight the dynamics of the particles spin on the unit sphere.

The parameters of the electromagnetic fields are the same as in Sec.5.1, with $E_{0}=E_{\text {ref }}$ and $\mathfrak{h}=0.00022$. In the figures 9 and 10 , we show the spins $\mathbf{s}$ of the electrons at different 


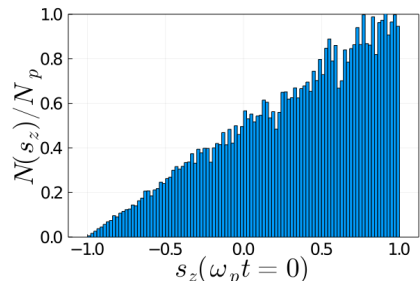

(a)

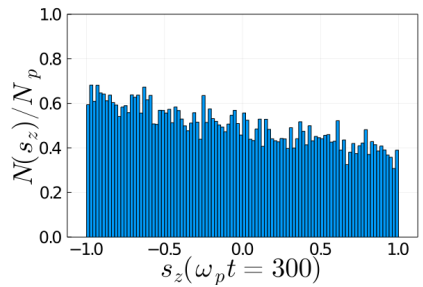

(d)

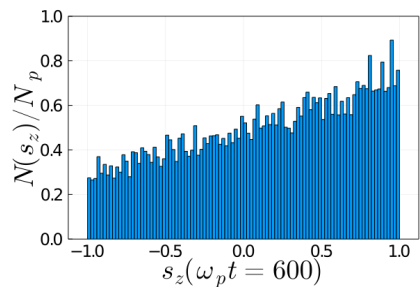

(g)

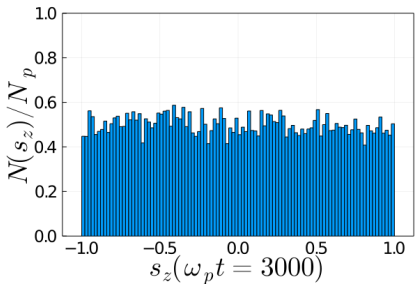

(j)

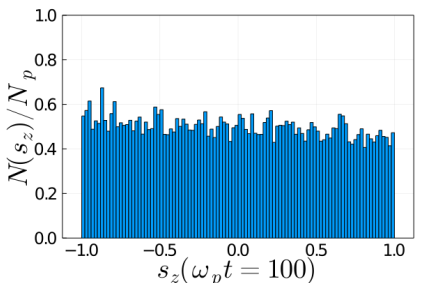

(b)

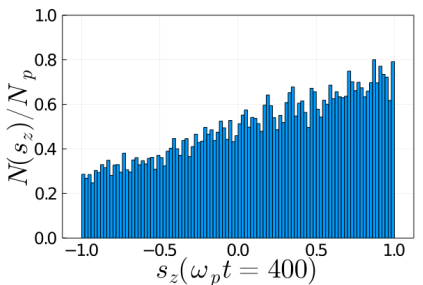

(e)

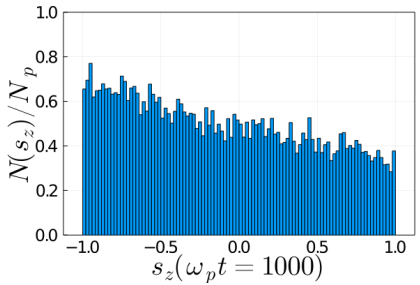

(h)

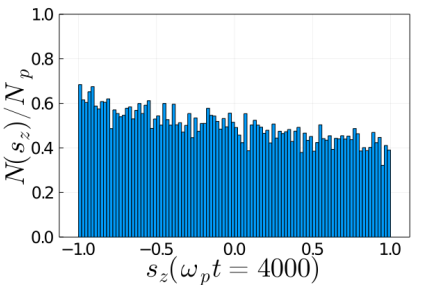

$(\mathrm{k})$

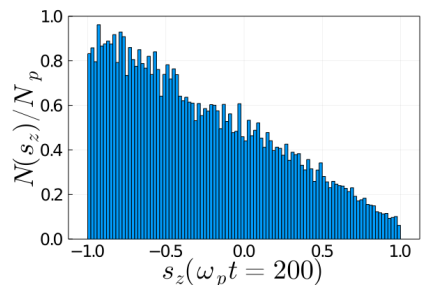

(c)

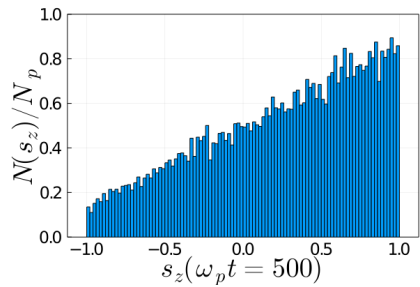

(f)

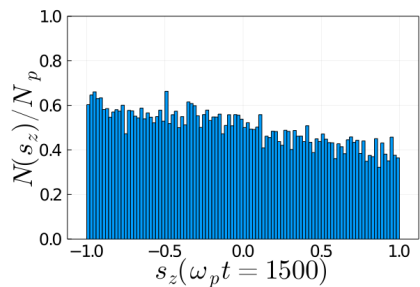

(i)

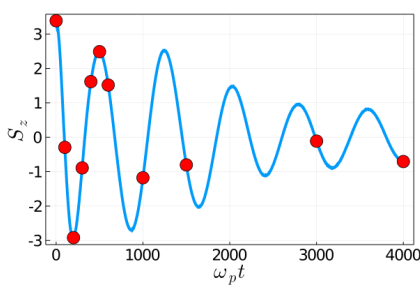

(l)

Figure 8. Full spin dependent model. Histograms of $s_{z}$ at different times, for a case with $\mathfrak{h}=0.00022, v_{t h}=0.17, \eta=1$. From (a) to (k): $\omega_{p} t=0,100,200,300,400,500,600,1000,1500,3000$, and $4000 . N\left(s_{z}\right)$ the number of particles having the $z$ spin component in an interval around $s_{z}$ and $N_{p}$ is the total number of particles. The frame (l) shows the time history of the global spin component $S_{z}(t)$. The red dots corresponds to the times of the different histograms displayed in (a)-(k).

instants $t$, together with the time history of the global spin components $S_{y}(t)$ and $S_{z}(t)$, for two values of the thermal speed $v_{t h}=0.17$ (figure 9 ) and $v_{t h}=0.51$ (figure 10). At $t=0$, the spin variables of all particles are localized at the north pole of the sphere, as indicated by the single red dot in the initial condition.

First, we remark that the spin vector of each particle remains on the unit sphere: $\left|\mathbf{s}_{a}(t)\right|=1, \forall a=1, \ldots, N_{p}$ and $\forall t>0$. This is of course the case for the continuous model, but it is worth emphasizing that our numerical scheme is capable of capturing this important geometric property exactly.

At later times, the spin distribution broadens and explores most of the surface of 
the sphere. For $v_{t h}=0.17$, the distribution moves around the surface of the sphere but remains rather localized on it. This is in line with the evolution of the global spin components $S_{y, z}(t)$ [figure $9(\mathrm{j})$-(k)], which oscillate with little damping. For $v_{t h}=0.51$, the distribution covers the surface of the sphere more uniformly, although the motion is not completely ergodic, and some ring-shaped structures are still visible at times $\omega_{p} t \gtrsim$ 1000. This is also consistent with the corresponding evolution of the spin components $S_{y, z}(t)$ [figure $10(\mathrm{j})-(\mathrm{k})$ ], which are more heavily damped than in the low-temperature case.

\section{Conclusion}

Spin effects in plasmas have been studied extensively during the last two decades (for a recent review, see (Manfredi et al. 2019)). From an experimental point of view, polarized electron beams can now be created and precisely manipulated in the laboratory ( $\mathrm{Wu}$ et al. 2019, 2020, Nie et al. 2021). Most theoretical works report on various analytical developments aimed at including ever more sophisticated effects, such as relativistic corrections or spin-orbit coupling. However, hardly any efforts have been devoted to the development of numerical methods that are suitable for simulating spin-polarized plasmas in realistic situations. The main objective of the present study was to implement an accurate numerical code for plasmas where the spin of the electrons plays a nonnegligible role.

Just like for ordinary plasmas, numerical methods for spin-polarized plasmas also come in two main families: Eulerian (grid-based) and Lagrangian (particle-based). This distinction is even more pertinent for spin plasmas. Indeed, the relevant quantum evolution equations can be formulated in terms of a Wigner distribution function, characterizing a quantum state in the classical phase space. The Wigner function for a particle with spin can be written either as a $2 \times 2$ matrix in the ordinary phase space $(\mathbf{x}, \mathbf{p})$ or, equivalently, as a scalar function in an extended phase space $(\mathbf{x}, \mathbf{p}, \mathbf{s})$ where the spin $\mathbf{s}$ is now an independent variable. Naturally, Eulerian methods are better adapted to the matrix form of the Wigner distribution function, whereas PIC methods are more suitable for the extended phase space representation (Manfredi et al. 2019).

In the semiclassical limit, the Wigner equation becomes a Vlasov-like equation that incorporates spin effects. In this approximation, the electron motion is described by classical trajectories, while the spin is treated as a fully quantum variable.

Here, we have developed a geometric PIC method for the spin Vlasov-Maxwell system, based on a noncanonical Hamiltonian structure (Marklund \& Morrison 2011) that preserves some of the main properties of the continuous equation (Kraus et al. 2017). This approach is coupled to a Hamiltonian splitting for the time discretization, which ensures long-time robustness, very good total energy conservation, and exact preservation of certain invariants (Poisson constraint or $\left|\mathbf{s}_{a}\right|=1$ ).

The PIC code has been tested on a standard laser-plasma problem, namely the stimulated Raman scattering of an electromagnetic wave interacting with an underdense plasma. In this case, the electrons of the plasma are spin-polarized, with different degrees of polarization. We have studied the influence of several physical parameters (temperature, electromagnetic field amplitude, quantum effects,...) on the Raman instability. The main result is that an initially polarized electron gas can lose its polarization through a combination of thermal effects and the Raman instability. These results may be interesting for current and future laser-plasma experiments that make use of polarized electron beams ( $\mathrm{Wu}$ et al. 2020; Nie et al. 2021), and also in condensed matter physics 


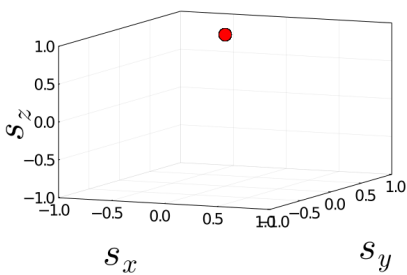

(a)

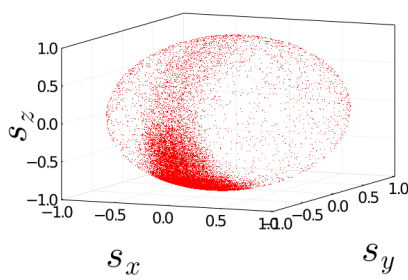

(d)

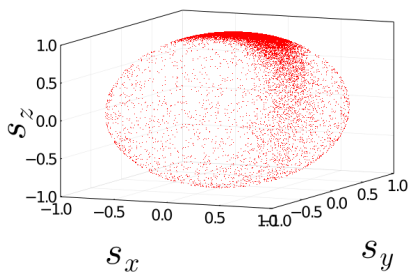

(g)

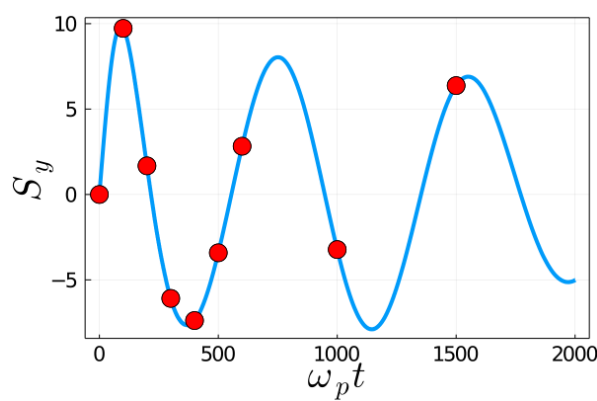

$(\mathrm{j})$

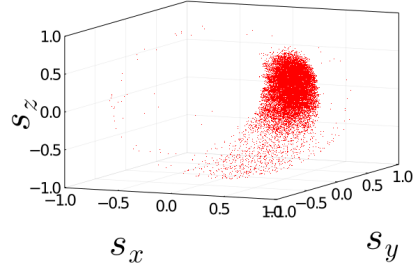

(b)

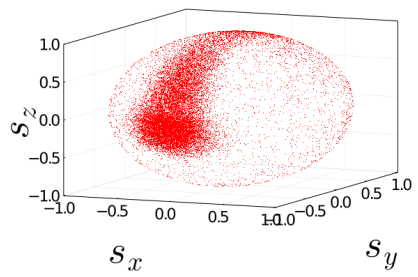

(e)

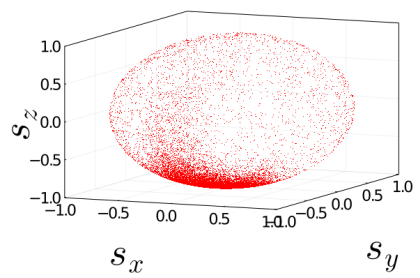

(h)

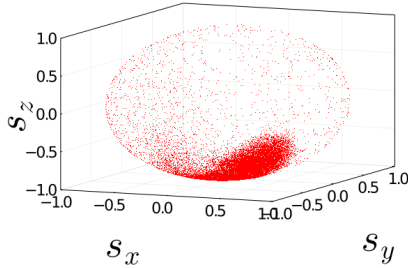

(c)

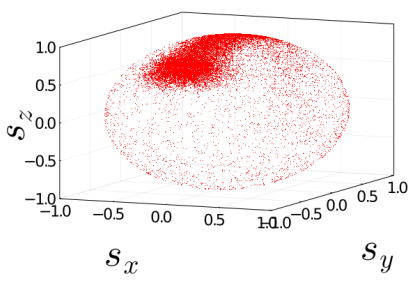

(f)

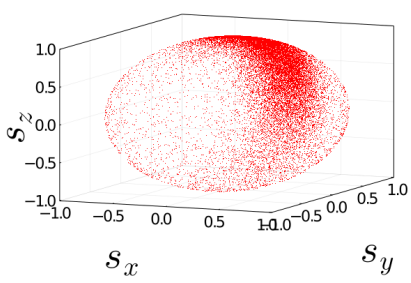

(i)

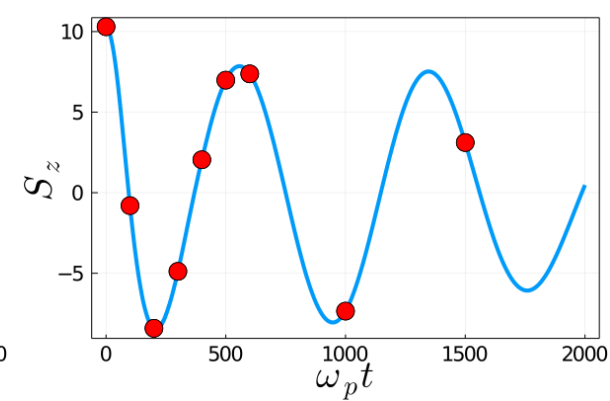

$(\mathrm{k})$

Figure 9. Full spin dependent model, with Dirac initial condition and $v_{t h}=0.17$. Frames from (a) to (i): distribution of the spins on the unit sphere, at times $\omega_{p} t=0,100,200,300,400,500,600,1000$, and 1500. Frames $(\mathrm{j})$ and $(\mathrm{k})$ show time history of $S_{y}(t)$ and $S_{z}(t)$, the red dots correspond to the times of the different snapshots displayed in (a)-(i).

for the understanding of the ultrafast demagnetization observed in magnetic materials irradiated with femtosecond laser pulses (Bigot \& Vomir 2013).

\section{Acknowledgements}

The authors wish to thank Pierre Navaro (CNRS, Rennes) for his help in the code development. Much of this work has been done during the one year stay of the author 


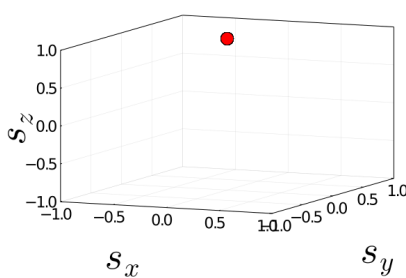

(a)

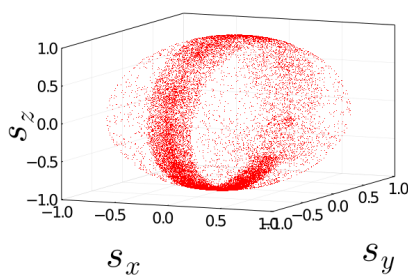

(d)

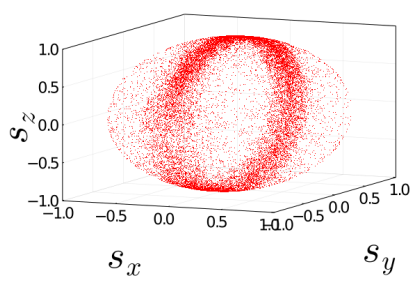

$(\mathrm{g})$

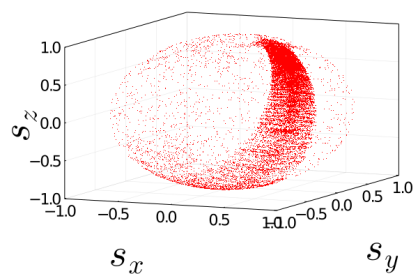

(b)

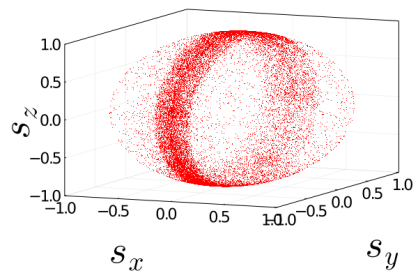

(e)

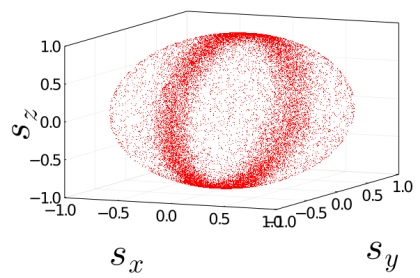

(h)

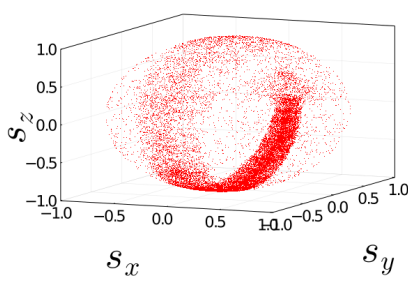

(c)

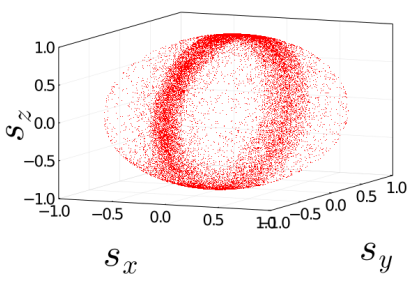

(f)

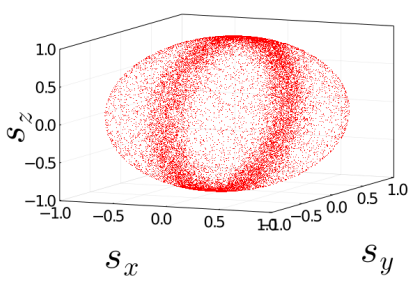

(i)

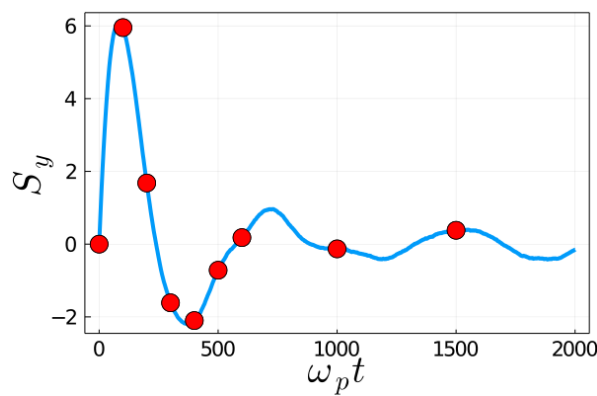

(j)

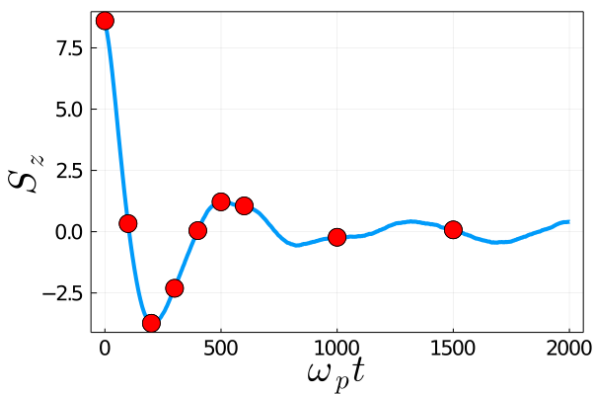

$(\mathrm{k})$

Figure 10. Full spin dependent model, with Dirac initial condition and $v_{t h}=0.51$. Frames from (a) to (i): distribution of the spins on the unit sphere, at times $\omega_{p} t=0,100,200,300,400,500,600,1000$, and 1500. Frames $(\mathrm{j})$ and $(\mathrm{k})$ show time history of $S_{y}(t)$ and $S_{z}(t)$, the red dots correspond to the times of the different snapshots displayed in (a)-(i).

Y. L. in Rennes which was supported by a scholarship from Academy of Mathematics and Systems Science, Chinese Academy of Sciences. Y. S. is supported by the National Natural Science Foundation of China No. 11771436. 


\section{Appendix A. Computation of the functional derivatives}

In this Appendix, we give some details on the discrete functional derivatives. We also refer to Kraus et al. (2017) for a more general context. The main point is to consider any functional $\mathcal{F}$ of the semi-discretized unknown $E_{x, h}, E_{y, h}, \ldots$ as a function $F$ of the coefficients $\mathbf{e}_{x}, \mathbf{e}_{y}, \ldots$ Thus, as $E_{x, h}(t)=\sum_{i=1}^{N_{1}} e_{x, i}(t) \Lambda_{i}^{1}(x)$, any functional $\mathcal{F}\left[E_{x, h}\right]$ will be considered as a function $F\left(\mathbf{e}_{x}\right)$. We then have the discrete functional derivatives by using the calculations in Kraus et al. (2017),

$$
\frac{\delta \mathcal{F}\left[E_{x, h}\right]}{\delta E_{x}}=\sum_{i=1}^{N_{1}} \frac{\partial F\left(\mathbf{e}_{x}\right)}{\partial e_{x, i}} \Sigma_{i}^{1}(x)=\sum_{i, j=1}^{N_{1}} \frac{\partial F\left(\mathbf{e}_{x}\right)}{\partial e_{x, i}}\left(\mathbf{M}_{1}^{-1}\right)_{i j} \Lambda_{j}^{1}(x) .
$$

Similarly, for the other fields, we have

$$
\begin{aligned}
& \frac{\delta \mathcal{F}\left[E_{y, h}\right]}{\delta E_{y}}=\sum_{i=1}^{N_{0}} \frac{\partial F\left(\mathbf{e}_{y}\right)}{\partial e_{y, i}} \Sigma_{i}^{0}(x)=\sum_{i, j=1}^{N_{0}} \frac{\partial F\left(\mathbf{e}_{y}\right)}{\partial e_{y, i}}\left(\mathbb{M}_{0}^{-1}\right)_{i j} \Lambda_{j}^{0}(x), \\
& \frac{\delta \mathcal{F}\left[E_{z, h}\right]}{\delta E_{z}}=\sum_{i=1}^{N_{0}} \frac{\partial F\left(\mathbf{e}_{z}\right)}{\partial e_{z, i}} \Sigma_{i}^{0}(x)=\sum_{i, j=1}^{N_{0}} \frac{\partial F\left(\mathbf{e}_{z}\right)}{\partial e_{z, i}}\left(\mathbb{M}_{0}^{-1}\right)_{i j} \Lambda_{j}^{0}(x), \\
& \frac{\delta \mathcal{F}\left[A_{y, h}\right]}{\delta A_{y}}=\sum_{i=1}^{N_{0}} \frac{\partial F\left(\mathbf{a}_{y}\right)}{\partial a_{y, i}} \Sigma_{i}^{0}(x)=\sum_{i, j=1}^{N_{0}} \frac{\partial F\left(\mathbf{a}_{y}\right)}{\partial a_{y, i}}\left(\mathbb{M}_{0}^{-1}\right)_{i j} \Lambda_{j}^{0}(x), \\
& \frac{\delta \mathcal{F}\left[A_{z, h}\right]}{\delta A_{z}}=\sum_{i=1}^{N_{0}} \frac{\partial F\left(\mathbf{a}_{z}\right)}{\partial a_{z, i}} \Sigma_{i}^{0}(x)=\sum_{i, j=1}^{N_{0}} \frac{\partial F\left(\mathbf{a}_{z}\right)}{\partial a_{z, i}}\left(\mathbb{M}_{0}^{-1}\right)_{i j} \Lambda_{j}^{0}(x) .
\end{aligned}
$$

Regarding the distribution function $f$, we assume a particle-like distribution function for $N_{p}$ particles

$$
f_{h}(x, p, \mathbf{s}, t)=\sum_{a=1}^{N_{p}} \omega_{a} \delta\left(x-x_{a}(t)\right) \delta\left(p-p_{a}(t)\right) \delta\left(\mathbf{s}-\mathbf{s}_{a}(t)\right)
$$

And a functional of the distribution function $\mathcal{F}[f]$ can be considered as a function of the particle phase space trajectories $F(\mathbf{X}, \mathbf{P}, \mathbf{S})$. From Kraus et al. $(2017)$, we have

$$
\frac{\partial F}{\partial x_{a}}=\left.\omega_{a} \frac{\partial}{\partial x} \frac{\delta \mathcal{F}}{\delta f}\right|_{\left(x_{a}, p_{a}, \mathbf{s}_{a}\right)}, \frac{\partial F}{\partial p_{a}}=\left.\omega_{a} \frac{\partial}{\partial p} \frac{\delta \mathcal{F}}{\delta f}\right|_{\left(x_{a}, p_{a}, \mathbf{s}_{a}\right)}, \frac{\partial F}{\partial \mathbf{s}_{a}}=\left.\omega_{a} \frac{\partial}{\partial \mathbf{s}_{a}} \frac{\delta \mathcal{F}}{\delta f}\right|_{\left(x_{a}, p_{a}, \mathbf{s}_{a}\right)} .
$$

\section{Appendix B. Proof of Theorem 1}

This section is devoted to the proof of Theorem 1. Since the matrix (3.14) is clearly skew symmetric, we only need to verify the Jacobi identity (the dependence on $\mathbf{u}$ of $\mathbb{J}$ will be omitted for clarity)

$$
\sum_{\ell}\left(\frac{\partial \mathbb{J}_{i j}}{\partial u_{\ell}} \mathbb{J}_{\ell k}+\frac{\partial \mathbb{J}_{j k}}{\partial u_{\ell}} \mathbb{J}_{\ell i}+\frac{\partial \mathbb{J}_{k i}}{\partial u_{\ell}} \mathbb{J}_{\ell j}\right)=0, \text { for all } i, j, k \text {. }
$$

As the Poisson matrix $\mathbb{l}=\mathbb{I}(\mathbf{u})$ depends only on $\mathbf{X}$ and $\mathbf{S}$, we only need to sum $\ell$ over $1 \leqslant \ell \leqslant N_{p}$ and $2 N_{p}+1 \leqslant \ell \leqslant 5 N_{p}$. There are two cases we need to consider.

- Two of $i, j, k \in \mathbb{Z}$ lie in $\left[N_{p}+1,2 N_{p}\right]$, and the other one lies in $\left[5 N_{p}+1,5 N_{p}+N_{1}\right]$. We take the case that $N_{p}+1 \leqslant i, j \leqslant 2 N_{p}$ and $5 N_{p}+1 \leqslant k \leqslant 5 N_{p}+N_{1}$ for example. In 
this case, Eq. (B 1) becomes

$$
\sum_{\ell=1}^{N_{p}}\left(\frac{\partial \rrbracket_{j k}}{\partial u_{\ell}} \rrbracket_{\ell i}+\frac{\partial \rrbracket_{k i}}{\partial u_{\ell}} \rrbracket_{\ell j}\right)=0 .
$$

As $\mathbb{J}_{j k}$ in the above depends only on $x_{j-N_{p}}, \mathbb{J}_{k i}$ depends only on $x_{i-N_{p}}$, the left hand side of the above identity becomes

$$
\frac{\partial \mathbb{J}_{j k}}{\partial u_{j-N_{p}}} \rrbracket_{\left(j-N_{p}\right) i}+\frac{\partial \mathbb{J}_{k i}}{\partial u_{i-N_{p}}} \rrbracket_{\left(i-N_{p}\right) j} .
$$

When $i \neq j, \mathbb{J}_{\left(j-N_{p}\right) i}=0$, and $\mathbb{J}_{\left(i-N_{p}\right) j}=0$, then the quantity $\mathrm{B} 2$ is zero. When $i=j$, we have $\mathbb{J}_{\left(j-N_{p}\right) i}=\mathbb{J}_{\left(i-N_{p}\right) j}$, and $\frac{\partial \unlhd_{j k}}{\partial u_{j-N_{p}}}=-\frac{\partial \unlhd_{k i}}{\partial u_{i-N_{p}}}$, then the quantity $\mathrm{B} 2$ is also zero which ends the proof.

- When $2 N_{p}+1 \leqslant i, j, k \leqslant 5 N_{p}$, we only need to sum $\ell$ over $2 N_{p}+1 \leqslant \ell \leqslant 5 N_{p}$. In this case, (B1) is easy to verify.

\section{Appendix C. Derivation of the spin precession frequency}

We consider the following ODE system

$$
\dot{\mathbf{S}}(t)=\mathbf{S}(t) \times \mathbf{B}(t, x), \quad s(t=0)=(0,1,0),
$$

with $\mathbf{B}(t, x)=\left(0, B_{0} \sin \left(\omega_{0} t\right),-B_{0} \cos \left(\omega_{0} t\right)\right)$ (which corresponds to a circularly-polarized incident wave). With the normalization $\bar{t}=B_{0} t$ and $\varepsilon=B_{0} / \omega_{0}$ which is assumed to be small, we obtain the following system

$$
\left\{\begin{array}{l}
\dot{S}_{x}(\bar{t})=-S_{y}(\bar{t}) \cos (\bar{t} / \varepsilon)-S_{z}(\bar{t}) \sin (\bar{t} / \varepsilon) \\
\dot{S}_{y}(\bar{t})=S_{x}(\bar{t}) \cos (\bar{t} / \varepsilon) \\
\dot{S}_{z}(\bar{t})=S_{x}(\bar{t}) \sin (\bar{t} / \varepsilon)
\end{array}\right.
$$

The system can then be rewritten as $\dot{\mathbf{u}}=F(\bar{t} / \varepsilon, \mathbf{u})$, where $\mathbf{u}=\mathbf{S}$ and $F(\cdot, \mathbf{u})$ is $2 \pi$ periodic. Introducing the augmented unknown $U(\bar{t}, \tau=\bar{t} / \varepsilon)=\mathbf{u}(t)$, the system is recast into

$$
\partial_{t} U+\frac{1}{\varepsilon} \partial_{\tau} U=F(\tau, U)
$$

We can perform a decomposition of this PDE: $U(\bar{t}, \tau)=U_{0}(\bar{t})+U_{1}(\bar{t}, \tau)$ where $U_{0}(\bar{t})=$ $\Pi U(\bar{t}, \cdot)$, the operator $\Pi$ being the average on $[0,2 \pi]$. Inserting the decomposition into (C3) leads to

$$
\left\{\begin{array}{r}
\partial_{t} U_{0}=\Pi F\left(\cdot, U_{0}+U_{1}\right), \\
\partial_{t} U_{1}+\frac{1}{\varepsilon} \partial_{\tau} U_{1}=(I-\Pi) F\left(\cdot, U_{0}+\varepsilon U_{1}\right) .
\end{array}\right.
$$

The asymptotic limit $\varepsilon \rightarrow 0$ is obtained by inserting the approximation obtained from the second equation $U_{1}=\varepsilon \partial_{\tau}^{-1}(I-\Pi) F\left(\cdot, U_{0}\right)+\mathcal{O}\left(\varepsilon^{2}\right)$ into the first equation. It comes

$$
\partial_{t} U_{0}=\Pi F\left(\cdot, U_{0}+\varepsilon \partial_{\tau}^{-1}(I-\Pi) F\left(\cdot, U_{0}\right)\right)+\mathcal{O}\left(\varepsilon^{2}\right) .
$$

First, we compute $U_{1}$

$$
\begin{aligned}
U_{1} & =\varepsilon \partial_{\tau}^{-1}(I-\Pi) F\left(\cdot, U_{0}\right)+\mathcal{O}\left(\varepsilon^{2}\right) \\
& =\varepsilon(I-\Pi) \int_{0}^{\tau} F\left(s, U_{0}\right) \mathrm{d} s+\mathcal{O}\left(\varepsilon^{2}\right)=\varepsilon\left(\begin{array}{c}
-U_{0, y} \sin \tau+U_{0, z} \cos \tau \\
U_{0, x} \sin \tau \\
-U_{0, x} \cos \tau
\end{array}\right)+\mathcal{O}\left(\varepsilon^{2}\right) .
\end{aligned}
$$


Then, (C 4) becomes

$\partial_{t}\left(\begin{array}{c}U_{0, x} \\ U_{0, y} \\ U_{0, z}\end{array}\right)=\Pi\left(\begin{array}{c}-\left(U_{0, y}+\varepsilon U_{0, x} \sin \tau\right) \cos \tau-\left(U_{0, z}-\varepsilon U_{0, x} \cos \tau\right) \sin \tau \\ \left(U_{0, x}+\varepsilon\left(-U_{0, y} \sin \tau+U_{0, z} \cos \tau\right)\right) \cos \tau \\ \left(U_{0, x}+\varepsilon\left(-U_{0, y} \sin \tau+U_{0, z} \cos \tau\right)\right) \sin \tau\end{array}\right)=\left(\begin{array}{c}0 \\ \varepsilon / 2 U_{0, z} \\ -\varepsilon / 2 U_{0, y}\end{array}\right)$

The first component does not depend on time and the asymptotic model for the two last component $u(t)=\left(U_{0, y}(t), U_{0, z}(t)\right)^{T}$ then reads, in the original time variable $t=\bar{t} / B_{0}$

$$
\dot{u}(t)=\frac{\varepsilon B_{0}}{2} J u,
$$

with $J$ the symplectic matrix. The solution then reads (using $\varepsilon=B_{0} / \omega_{0}$ )

$$
u(t)=\exp \left(\frac{B_{0}^{2}}{2 \omega_{0}} t J\right) u(0)=\left(\begin{array}{cc}
\cos \left(B_{0}^{2} /\left(2 \omega_{0}\right) t\right) & \sin \left(B_{0}^{2} /\left(2 \omega_{0}\right) t\right) \\
-\sin \left(B_{0}^{2} /\left(2 \omega_{0}\right) t\right) & \cos \left(B_{0}^{2} /\left(2 \omega_{0}\right) t\right)
\end{array}\right) u(0),
$$

whose frequency is $\omega=\frac{B_{0}^{2}}{2 \omega_{0}}$.

\section{Appendix D. Spin-dependent initial condition}

The spin Vlasov model adopted here is a semiclassical approximation whereby the mechanical motion (position and momentum) is treated classically, while the spin degrees of freedom are fully quantum variables. Hence, the spin-dependent part of the initial distribution function should be determined using the rules of quantum mechanics for spin-1/2 fermions. We will do this by making use of Wigner functions (representation of quantum mechanics in the classical phase space). The derivation follows closely Manfredi et al. (2019).

For spin- $1 / 2$ particles, the relevant Wigner function $\mathcal{F}$ is a $2 \times 2$ matrix:

$$
\mathcal{F}=\left(\begin{array}{ll}
f^{\uparrow \uparrow} & f^{\uparrow \downarrow} \\
f^{\downarrow \uparrow} & f^{\downarrow \downarrow}
\end{array}\right)
$$

where the symbols $\uparrow, \downarrow$ denotes respectively the spin-up and spin-down components. It is convenient to project the matrix $\mathcal{F}$ onto the Pauli basis set

$$
\mathcal{F}=\frac{1}{2} \sigma_{0} F_{0}+\frac{1}{2} \mathbf{F} \cdot \sigma
$$

where $\sigma=\left(\sigma_{x}, \sigma_{y}, \sigma_{z}\right)$ is the vector of the $2 \times 2$ Pauli matrices

$$
\sigma_{x}=\left(\begin{array}{cc}
0 & 1 \\
1 & 0
\end{array}\right), \sigma_{y}=\left(\begin{array}{cc}
0 & -i \\
i & 0
\end{array}\right), \sigma_{z}=\left(\begin{array}{cc}
1 & 0 \\
0 & -1
\end{array}\right)
$$

$\sigma_{0}$ is the $2 \times 2$ identity matrix, and

$$
F_{0}=\operatorname{Tr}\{\mathcal{F}\}=f^{\uparrow \uparrow}+f^{\downarrow \downarrow}, \quad \mathbf{F}=\operatorname{Tr}(\mathcal{F} \sigma) .
$$

The scalar distribution function in the extended phase space $f(\mathbf{x}, \mathbf{p}, \mathbf{s})$ is related to the matrix Wigner function through Marklund et al. (2010); Manfredi et al. (2019):

$$
f(\mathbf{x}, \mathbf{p}, \mathbf{s})=\frac{1}{4 \pi} \sum_{\alpha, \beta=1}^{2}\left(1+\mathbf{s} \cdot \sigma_{\alpha \beta}\right) \mathcal{F}_{\beta \alpha}(\mathbf{x}, \mathbf{p})
$$

In the so-called collinear approximation, the spin is directed either parallel or antiparallel to the $z$ axis, so that only the diagonal terms in (D 1) survive. Using (D 4), we get: 
$F_{0}=f^{\uparrow \uparrow}+f^{\downarrow \downarrow}, F_{z}=f^{\uparrow \uparrow}-f^{\downarrow \downarrow}$, and $F_{x}=F_{y}=0$. Using these expressions, it is easy to show that

$$
f(\mathbf{x}, \mathbf{p}, \mathbf{s})=\frac{1}{4 \pi}\left(F_{0}+s_{z} F_{z}\right)
$$

For a Maxwell-Boltzmann equilibrium, $F_{0}$ is a standard Maxwellian distribution, and one can prove that $F_{z}(\mathbf{p})=\eta F_{0}(\mathbf{p})$, where $\eta \in[0,1]$ is a number that characterizes the degree of polarization of the electron gas (Manfredi et al. 2019). Hence, one can write:

$$
f(\mathbf{x}, \mathbf{p}, \mathbf{s})=\frac{1}{4 \pi}\left(1+\eta s_{z}\right) F_{0}(\mathbf{p}) .
$$

We also note that, using the distribution (D 6), the average values of the spin components are:

$$
\left\langle s_{x}\right\rangle=\left\langle s_{y}\right\rangle=0, \quad\left\langle s_{z}\right\rangle=\eta / 3 .
$$

The global spin components used in the main text are then: $S_{i}(t)=L\left\langle s_{i}\right\rangle$, with $i=$ $(x, y, z)$, where $L$ is the length of the periodic computational box.

\section{REFERENCES}

Arnold, A. \& Steinrück, H. 1989 The electromagnetic Wigner equation for an electron with spin. ZAMP Zeitschrift für angewandte Mathematik und Physik 40 (6), 793-815.

Asenjo, F., Zamanian, J., Marklund, M., Brodin, G. \& Johansson, P. 2012 Semirelativistic effects in spin-1/2 quantum plasmas. New J. Phys. 14 (7), 073042.

Beaurepaire, E., Merle, J., Daunois, A. \& Bigot, J. 1996 Ultrafast spin dynamics in ferromagnetic nickel. Phys. Rev. Lett. 76 (22), 4250-4253.

Bigot, J. \& VomiR, M. 2013 Ultrafast magnetization dynamics of nanostructures. Annalen der Physik 525, 2-30.

Bigot, J., Vomir, M. \& Beaurepaire, E. 2009 Coherent ultrafast magnetism induced by femtosecond laser pulses. Nat. Phys. 5 (7), 515-520.

Brodin, G., Holkundkar, A. \& Marklund, M. 2013 Particle-in-cell simulations of electron spin effects in plasmas. Journal of Plasma Physics 79 (4), 377-382.

Brodin, G., Marklund, M., Zamanian, J., Ericsson, A. \& Mana, P. L. 2008 Effects of the g-factor in semiclassical kinetic plasma theory. Phys. Rev. Lett. 101 (24), 245002.

Brodin, G., Marklund, M., Zamanian, J. \& Stefan, M. 2011 Spin and magnetization effects in plasmas. Plasma Physics and Controlled Fusion 53 (7), 074013.

Brodin, G. \& Stefan, M. 2013 Kinetic theory of fully degenerate electrons in the long scale limit. Phys. Rev. E 88, 023107.

Burby, J. 2017 Finite-dimensional collisionless kinetic theory. Phys. Plasmas 24, 032101.

Casas, F., Crouseilles, N., Faou, E. \& Mehrenberger, M. 2017 High-order hamiltonian splitting for the Vlasov-Poisson equations. Numerische Mathematik 135 (3), 769-801.

Cowley, S. C., Kulsrud, R. M. \& Valeo, E. 1986 A kinetic equation for spin-polarized plasmas. The Physics of Fluids 29 (2), 430-441.

Crouseilles, N., Einkemmer, L. \& Faou, E. 2015 Hamiltonian splitting for the VlasovMaxwell equations. J. Comput. Phys. 283, 224-240.

Dauger, D., Decyk, V. \& Dawson, J. 2005 Using semiclassical trajectories for the timeevolution of interacting quantum-mechanical systems. Journal of Computational Physics 209 (2), 559-581.

Forslund, D., Kindel, J. \& Lindman, E. 1975 Theory of stimulated scattering processes in laser-irradiated plasmas. Phys. Fluid 18, 1002.

Ghizzo, A., Bertrand, P., Shoucri, M., Johnston, T., Fijalkow, E. \& Feix, M. 1990 A Vlasov code for the numerical simulation of stimulated Raman scattering. J. Comput. Phys. 90, 431-457.

Hairer, E., Lubich, C. \& Wanner, G. 2002 Geometric Numerical Integration: StructurePreserving Algorithms for Ordinary Differential Equations. In Springer Series in Computational Mathematics. Springer-Verlag, Berlin. 
He, Y., Qin, H., Sun, Y., Xiao, J., Zhang, R. \& Liu, J. 2015 Hamiltonian integration methods for Vlasov-Maxwell equations. Phys. Plasmas 22, 124503.

He, Y., Sun, Y., Qin, H. \& Liu, J. 2016 Hamiltonian particle-in-cell methods for VlasovMaxwell equations. Phys. Plasmas 23 (9), 092108.

Huot, F., Ghizzo, A., Bertrand, P., Sonnendrücker, E. \& Coulaud, O. 2003 Instability of the time splitting scheme for the one-dimensional and relativistic Vlasov-Maxwell system. J. Comput. Phys. 185 (2), 512-531.

Hurst, J., Hervieux, P.-A. \& Manfredi, G. 2017 Phase-space methods for the spin dynamics in condensed matter systems. Philos. Trans. R. Soc. A Math. Phys. Eng. Sci. 375, 20160199.

Hurst, J., Hervieux, P.-A. \& Manfredi, G. 2018 Spin current generation by ultrafast laser pulses in ferromagnetic nickel films. Phys. Rev. B 97, 014424.

Hurst, J., Morandi, O., Manfredi, G. \& Hervieux, P.-A. 2014 Semiclassical Vlasov and fluid models for an electron gas with spin effects. Eur. Phys. J. D 68 (6), 176.

Kraus, M., Kormann, K., Morrison, P. \& Sonnendrücker, E. 2017 GEMPIC: geometric electromagnetic Particle-In-Cell methods. J. of Plasma Phys. 83 (4), 905830401.

Krieger, K., Dewhurst, J., Elliott, P., Sharma, S. \& Gross, E. 2015 Laser-induced demagnetization at ultrashort time scales: predictions of TDDFT. J. Chem. Theory Comput. 11, 4870.

Li, F., Decyk, V. K., Miller, K. G., Tableman, A., Tsung, F. S., Vranic, M., Fonseca, R. A. \& MorI, W. B. 2021 Accurately simulating nine-dimensional phase space of relativistic particles in strong fields. Journal of Computational Physics 438, 110367.

Li, Y., He, Y., Sun, Y., Niesen, J., Qin, H. \& Liu, J. $2019 a$ Solving the Vlasov-Maxwell equations using Hamiltonian splitting. J. Comput. Phys. 396, 381-399.

Li, Y., Sun, Y. \& Crouseilles, N. 2020 Numerical simulations of one laser-plasma model based on Poisson structure. J. Comput. Phys. 405, 109172.

Manfredi, G., Hervieux, P.-A. \& Hurst, J. 2019 Phase-space modeling of solid-state plasmas - A journey from classical to quantum. Rev. Mod. Plasma Phys. 3, 13.

Marklund, M. \& Morrison, P. 2011 Gauge-free Hamiltonian structure of the spin MaxwellVlasov equations. Physics Letters A 375 (24), 2362-2365.

Marklund, M., Zamanian, J. \& Brodin, G. 2010 Spin kinetic theory - quantum kinetic theory in extended phase space. Transp. Theory Stat. Phys. 39 (5), 502-523.

Marsden, J. \& Weinstein, A. 1982 The Hamiltonian structure of the Maxwell-Vlasov equations. Physica D 4 (3), 394-406.

Mehdaoui, B., Tan, R., Meffre, A., Carrey, J., Lachaize, S., Chaudret, B. \& Respaud, M. 2013 Increase of magnetic hyperthermia efficiency due to dipolar interactions in lowanisotropy magnetic nanoparticles: Theoretical and experimental results. Phys. Rev. B 87, 174419.

Moldabekov, Z., Bonitz, M. \& Ramazanov, T. 2018 Theoretical foundations of quantum hydrodynamics for plasmas. Physics of Plasmas 25, 031903.

Morrison, P. 2017 Structure and structure-preserving algorithms for plasma physics. Physics of Plasmas 24, 055502.

Nie, Z., Li, F., Morales, F., Patchkovskit, S., Smirnova, O., An, W., Nambu, N., Matteo, D., Marsh, K. A., Tsung, F., Mori, W. B. \& Joshi, C. 2021 In Situ generation of high-energy spin-polarized electrons in a beam-driven plasma wakefield accelerator. Phys. Rev. Lett. 126, 054801.

Qin, H., Liu, J., XiaO, J., Zhang, R., He, Y., Wang, Y., Sun, Y., Burby, J., Ellison, L. \& Zhou, Y. 2015 Canonical symplectic Particle-In-Cell method for long-term large-scale simulations of the Vlasov-Maxwell equations. Nuclear Fusion 56 (1), 014001.

Tonge, J., Dauger, D. E. \& Decyk, V. K. 2004 Two-dimensional semiclassical particle-in-cell code for simulation of quantum plasmas. Computer physics communications 164 (1-3), 279-285.

Wen, M., Keitel, C. H. \& Bauke, H. 2017 Spin-one-half particles in strong electromagnetic fields: Spin effects and radiation reaction. Phys. Rev. A 95, 042102.

Wen, M., Tamburini, M. \& Keitel, C. H. 2019 Polarized laser-wakefield-accelerated kiloampere electron beams. Phys. Rev. Lett. 122, 214801.

Wu, Y., Ji, L., Geng, X., Thomas, J., Büscher, M., Pukhov, A., Hützen, A., Zhang, L., 
Shen, B. \& LI, R. 2020 Spin filter for polarized electron acceleration in plasma wakefields. Phys. Rev. Applied 13, 044064.

Wu, Y., Ji, L., Geng, X., Yu, Q., Wang, N., Feng, B., Guo, Z., Wang, W., Qin, C., Yan, X. \& ET AL. 2019 Polarized electron-beam acceleration driven by vortex laser pulse. New J. Phys. 11, 073052.

XiaO, J., Qin, H., Liu, J., He, Y., Zhang, R. \& Sun, Y. 2015 Explicit high-order non-canonical symplectic Particle-In-Cell algorithms for Vlasov-Maxwell systems. Phys. Plasmas 22 (11), 112504.

Zamanian, J., Marklund, M. \& Brodin, G. $2010 a$ Scalar quantum kinetic theory for spin-1/2 particles: mean field theory. New J. Phys. 12 (4), 043019.

Zamanian, J., Stefan, M., Marklund, M. \& Brodin, G. $2010 b$ From extended phase space dynamics to fluid theory. Phys. Plasmas 17 (10), 102109. 